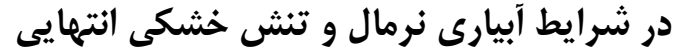

بيمان شريفى '، على اكبر عبادى ' و هاشم امين يناه

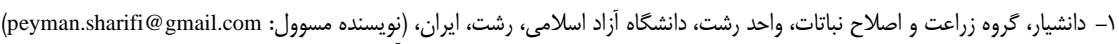

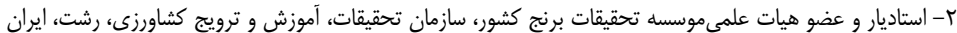

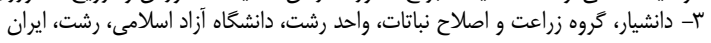

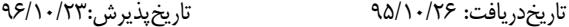

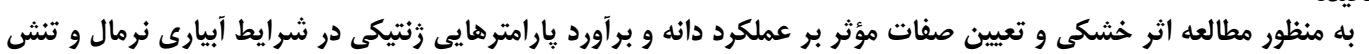

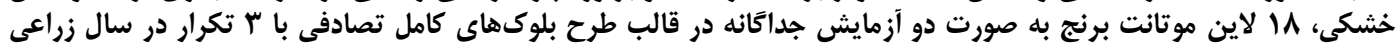

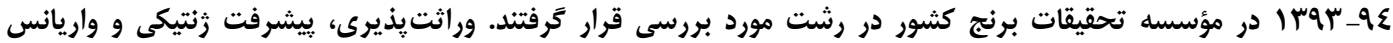

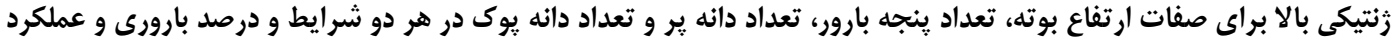

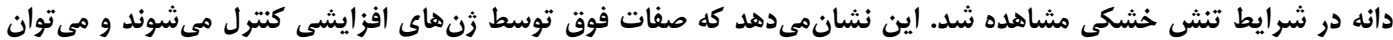

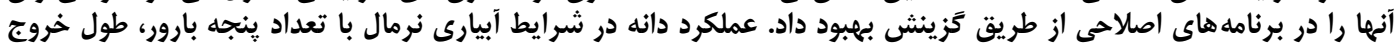

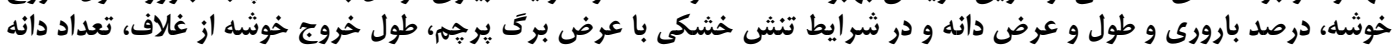

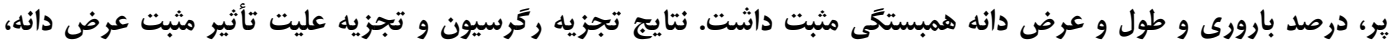

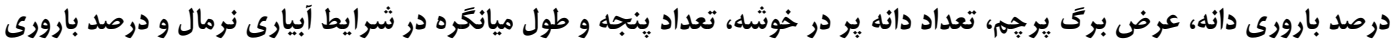

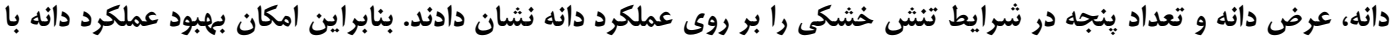

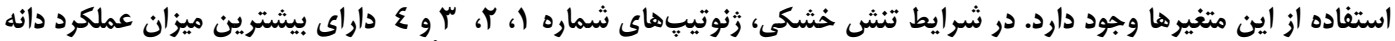

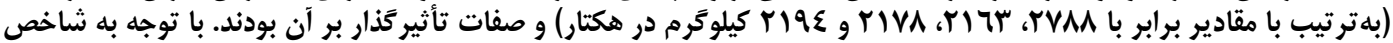

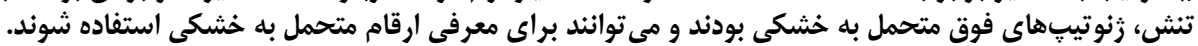

وازههاى كليدى: برنج، همبستكى، ركرسيون، كمرآبيارى، عليت، وراثت پِذيرى

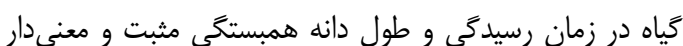

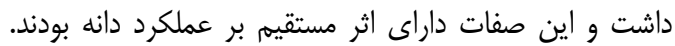

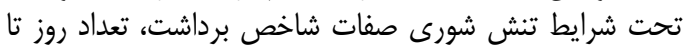

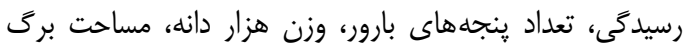

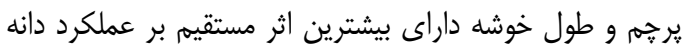

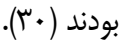

بررسى منابع نشان داد كه عملكرد دانه در شرايط غير

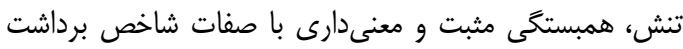

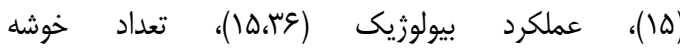

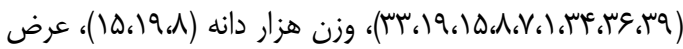

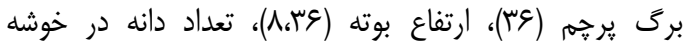

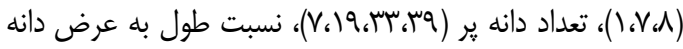

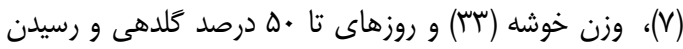

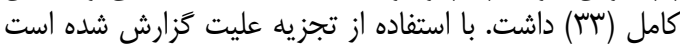

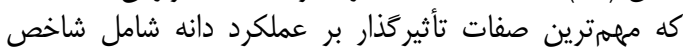

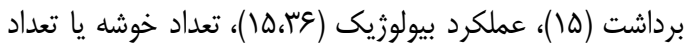

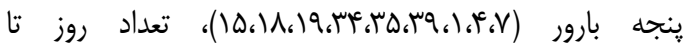

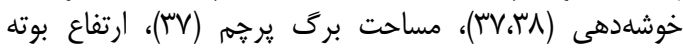

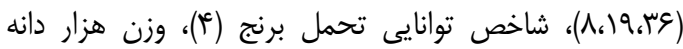

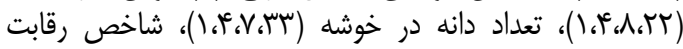

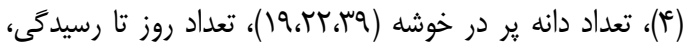

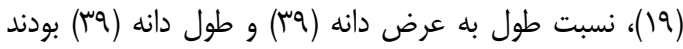
كه بيشترين اثر مستقيم را بر عملكرد دانه داشتند.
برنج دومين محصول استراتزيك كشور يس از گَندم

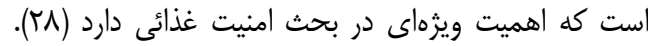

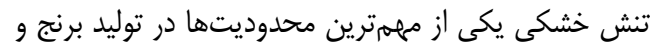

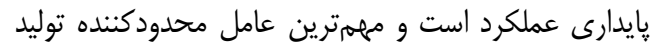

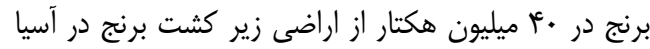

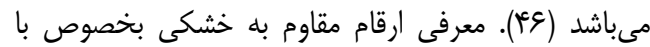

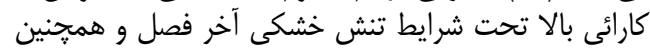

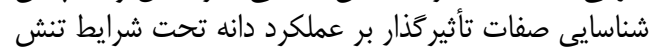

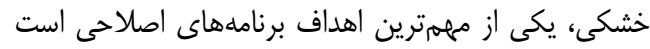

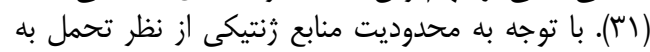

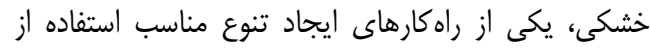

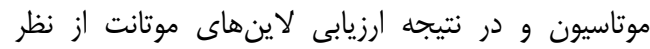

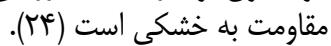

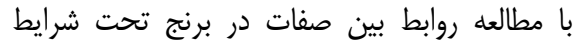

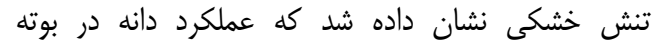

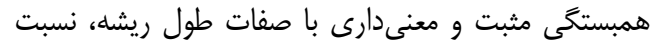

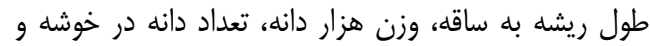

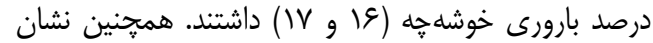

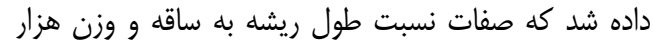

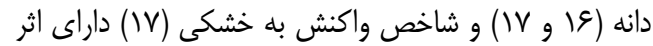

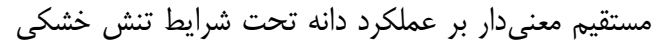

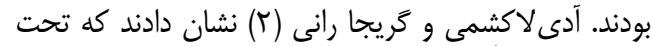

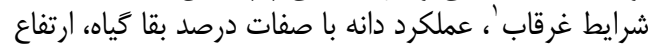




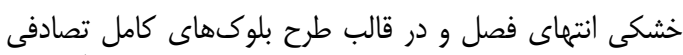

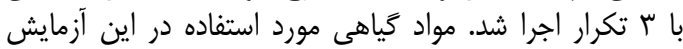

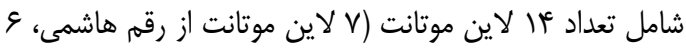

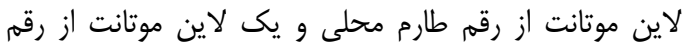

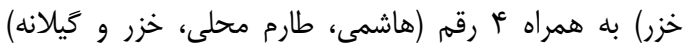

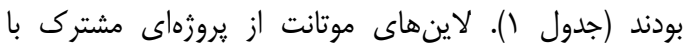

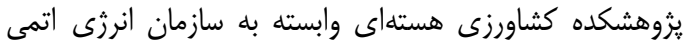

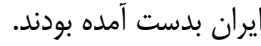

هدف از تحقيق حاضر بررسى اثرات مستقيه و و و

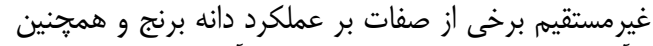

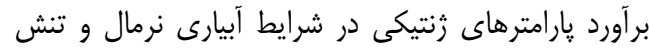

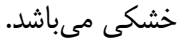

مواد و روشها

اين آزمايش در سال زراعى عأو-بوجا در مؤسسه

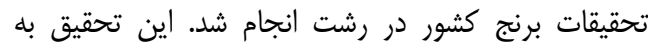

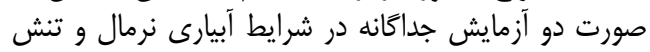

Table 1. Pedigree of rice mutant lines

جدول 1- شجره لاينهاى موتانت برنج

\begin{tabular}{|c|c|c|c|c|c|c|c|}
\hline \multicolumn{6}{|c|}{ شجره لاينهاى موتانت } & \multirow[b]{2}{*}{ كد لاين } & \multirow[b]{2}{*}{.$\hat{3}^{\circ}$} \\
\hline 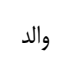 & 14199 & irq. & $\mid$ |rq & rat & 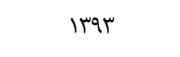 & & \\
\hline طارم & TM2-230-3 & TM3-230-VE-7 & TM4-230-VE-7 & TM5-230-VE-7 & TM6-230-VE-7 & TM6-230-VE-7-5-1 & 1 \\
\hline طَآرم & TM2-230-4 & TM3-230-VE-8 & $\begin{array}{c}\text { TM4-230-VE-8- } \\
4\end{array}$ & TM5-230-VE-8-4 & TM6-230-VE-8-4 & TM6-230-VE-8-4-1 & r \\
\hline طآرم & TM2-250-10 & TM3-250-10-7 & TM4-250-10-7-1 & TM5-250-10-7-1 & TM6-250-10-7-1 & TM6-250-10-7-1 & r \\
\hline طَارم & TM2-B-2 & TM3-B-2-1 & TM4-B-2-1-E & TM5-B-2-1-E & TM6-B-2-1-E & TM6-B-2-1-E & f \\
\hline طَآرم & TM2-B-7 & TM3-B-7-1 & TM4-B-7-1 & TM5-B-7-1 & TM6-B-7-1 & TM6-B-7-1 & $\Delta$ \\
\hline ط طآرم & TM2-B-19 & TM3-B-19-2 & TM4-B-19-2 & TM5-B-19-2 & TM6-B-19-2 & TM6-B-19-2 & 8 \\
\hline هاشمى & HM1-250-E-1 & HM2-250-E-1-1 & HM3-250-E-1-1 & HM4-250-E-1-1 & HM5-250-E-1-1 & HM5-250-E-1-1 & $v$ \\
\hline هاشمى & HM1-250-E-3 & HM2-250-E-3-2 & HM3-250-E-3-2 & HM4-250-E-3-2 & HM5-250-E-3-2 & HM5-250-E-3-2 & $\wedge$ \\
\hline هاشمى & HM1-250-7 & HM2-250-7-1 & HM3-250-7-1 & HM4-250-7-1 & HM5-250-7-1 & HM5-250-7-1 & 9 \\
\hline هاشمى & HM1-250-7 & HM2-250-7-6 & HM3-250-7-6 & HM4-250-7-6 & HM5-250-7-6 & HM5-250-7-6 & 1. \\
\hline هاشمى & HM1-300-E & HM2-300-E-1 & HM3-300-E-1 & HM4-300-E-1 & HM5-300-E-1 & HM5-300-E-1 & 11 \\
\hline 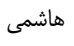 & HM1-300-3 & HM2-300-3-1 & HM3-300-3-1 & HM4-300-3-1 & HM5-300-3-1 & HM5-300-3-1 & ir \\
\hline هاشمى & HM1-300-5 & HM2-300-5-1 & HM3-300-5-1 & HM4-300-5-1 & HM5-300-5-1 & HM5-300-5-1 & rו \\
\hline خزر & KM1-200-4 & KM2-200-4-2 & КM3-200-4-2-E & KM4-200-4-2-E & KM5-200-4-2-E & KM5-200-4-2-E & if \\
\hline \multicolumn{7}{|c|}{ 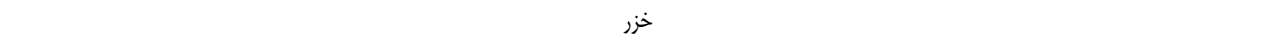 } & 10 \\
\hline \multicolumn{7}{|c|}{ هاشمى } & 19 \\
\hline \multicolumn{7}{|c|}{ طارم } & IV \\
\hline \multicolumn{7}{|c|}{ كيلانه } & $M$ \\
\hline
\end{tabular}

حرف T نشاندهنده طارم محلى، حرف H نشاندهنده هاشمى، حرف K نشاندهنده خزر، حرف M نشاندهنده موتاسيون و عدد بعد از حرف M نشاندهنده نسل موتاسيون

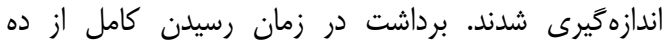

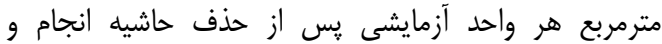

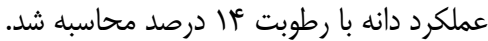
واريانسهاى فنوتييى ( $)$

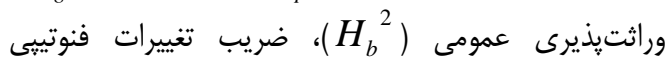
(PCV)

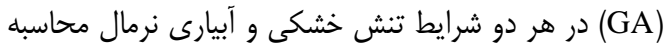

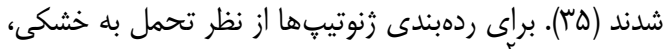

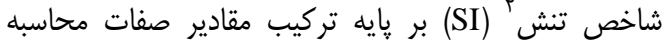

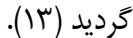

در آزمايش مربوط به تنش خشازيى انتهاى فصل،

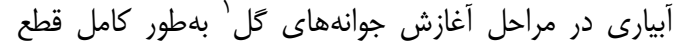

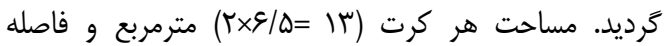

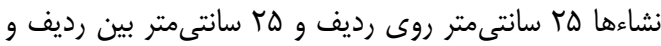

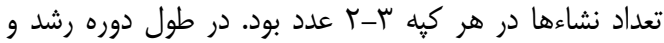

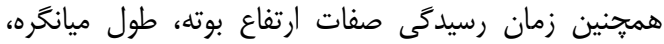

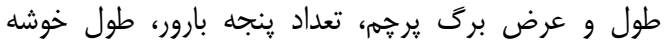

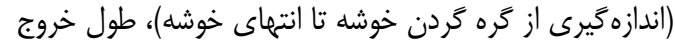
خوشه از غلاف (اندازهگيرى از محرد

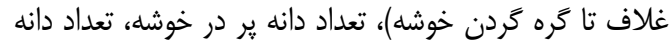

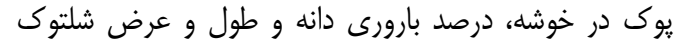


در اكثر صفات مورد مطالعه در هر دو شرايط نشان داد كه

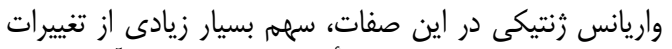

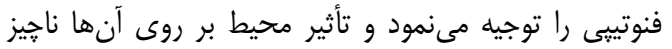

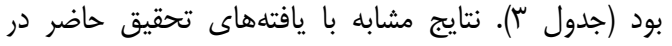

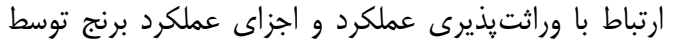

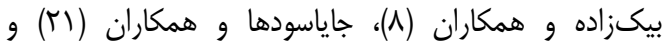

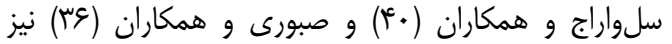
كزارش شده است. با توجه به اينكه وجود وراثتيذيرى بارئ الا فقط نشاندهنده

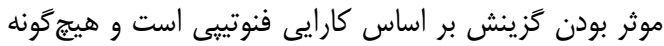

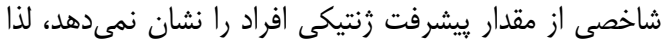

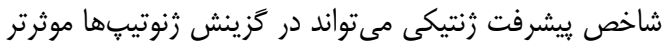

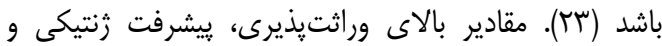

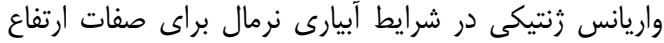

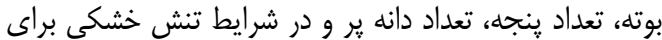

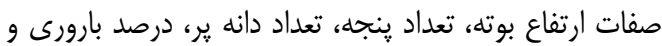

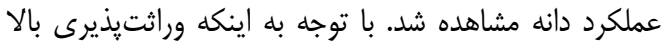

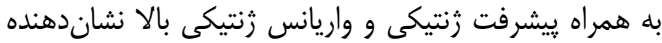

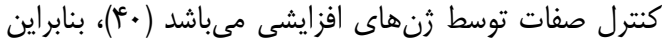

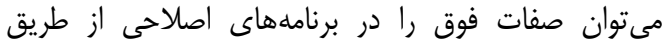

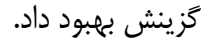
تجزيه همبستكى ترئى

نتايج تجزيه همبستخى نشيسى نشان داد كه در شرايط آبيارى

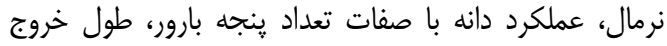

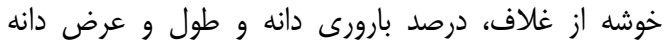

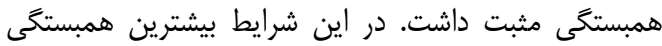

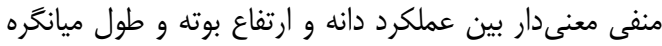

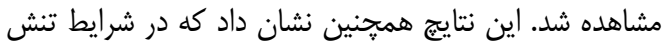

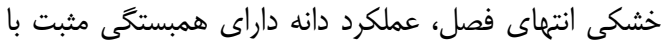

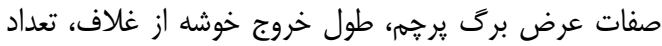

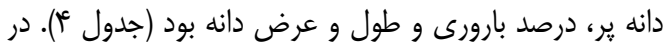

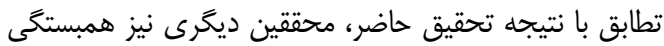

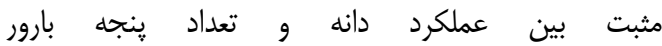

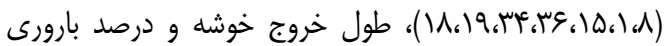

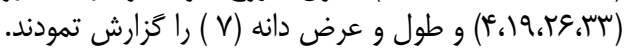

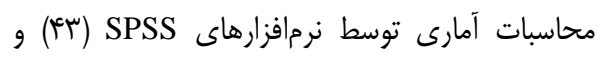
عاليت Path analysis

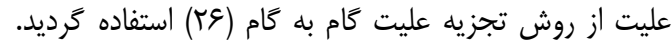

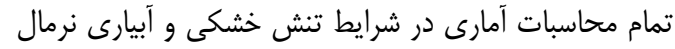
به صورت مجزا انجام گرفتند.

نتايج و بحث

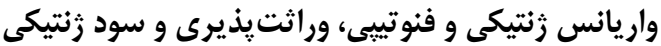

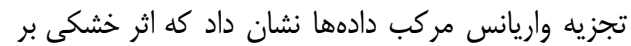

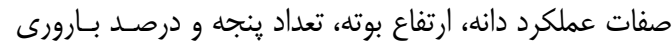

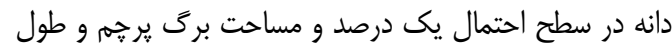

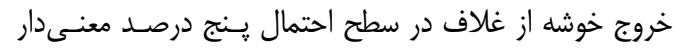

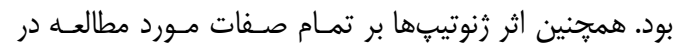

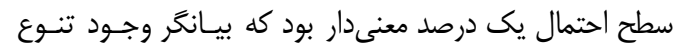

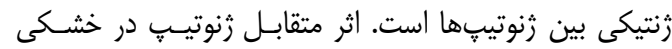

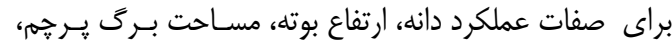

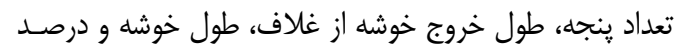

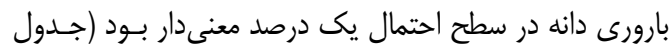

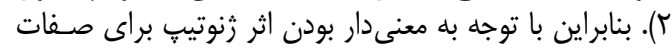

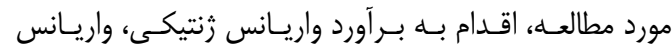

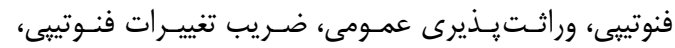

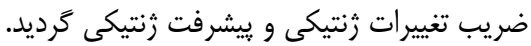

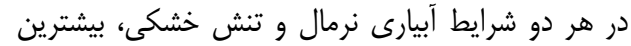

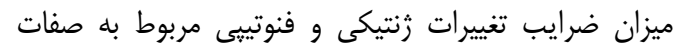

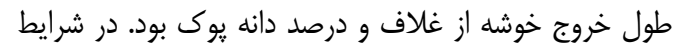

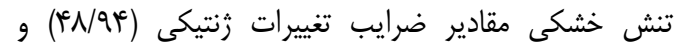

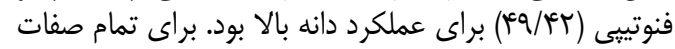

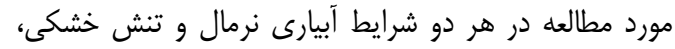

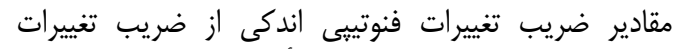

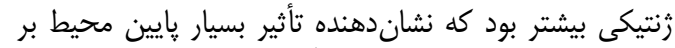

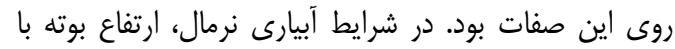

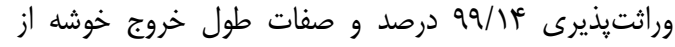
غلاف و نسبت طول به عرض دانه با وراثتيذيرى حدون خدود خرو

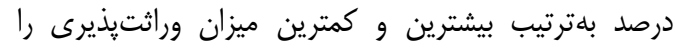

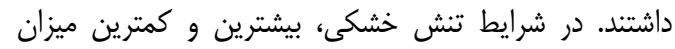

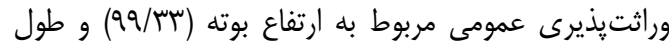

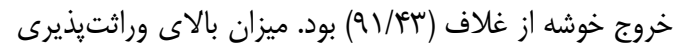


جدول r- تجزيه واريانس مركب عملكرد دانه و برخى از صفات مورفولوزيكى برنج در شرايط تنش خشكى و آبيارى نرمال Table 2. Combined analysis of variance for grain yield and some of the rice morphological trtaits under drought stress

\begin{tabular}{|c|c|c|c|c|c|c|c|c|c|}
\hline \multicolumn{8}{|c|}{ ميانكين مربعات } & \multirow[b]{2}{*}{ درجه آزادى } & \multirow[b]{2}{*}{ منابع تغييرات } \\
\hline نسبت طول به عرض دانه & درصد بارورى دانه & طول خروج خوشه از & طول خوشه & تعداد קينجه & مساحت برى يرجم & ارتفاع بوته & عملكرد دانه & & \\
\hline.$/ r^{\mathrm{ns}}$ & $m e F / g r^{* *}$ & $q \pi / \wedge s^{*}$ & $\Delta / \Delta \Delta^{\mathrm{ns}}$ & $\mid V g / \psi^{* *}$ & $\mid K F / \Psi^{*}$ & $0.9 \mathrm{~V} / \mathrm{\Lambda})^{* *}$ & $191 \wedge 1 \wedge \mathrm{V} \cdot 9^{* * *}$ & 1 & خشكى \\
\hline$\cdot / \cdot v$ & $\Delta \backslash / T)$ & $\Delta / \Lambda$. & $1 / \mu e$ & $1 / 4$ & 1 I/qT & $I V / V T$ & $r \Delta . .9 q / \Delta$ & f & تكرار درون خشكى \\
\hline.$/ 4^{* * *}$ & $\operatorname{Fq} / 19^{* *}$ & $r / \mu r^{* *}$ & $r q / \mu^{* * *}$ & $r q / \Delta \Delta^{* *}$ & $v \cdot / q r^{* *}$ & $1.11 / 1 e^{* *}$ & $\Lambda r \cdot \Lambda r q / \Lambda^{* *}$ & IV & رنوتيب \\
\hline$\cdot 110^{\mathrm{ns}}$ & $\pi \cdot / M^{* *}$ & $r / r \omega^{* *}$ & $r / r^{* *}$ & $r / r I^{* *}$ & ( & $19 \Delta / \Delta T^{* *}$ & $\mu F I \cdot r V / \Lambda^{* *}$ & iv & ثرنوتيب × خشكى \\
\hline.$/ 19$ & $r \Delta / \Lambda$. & $1 / 1 \mu$ & $1 / r q$ & r & $r / 9 \varphi$ & $9 / \mp q$ & $r V \omega \phi F / V$ & 91 & خطا \\
\hline 1.1 .9 & STr & $|N / \mu|$ & F/T & $\mid r / \Delta T$ & $q / \cdot r$ & $r / T$. & $8 / 1 f$ & & ضريب تغييرات \\
\hline
\end{tabular}

جدول ץ- يارامترهاى زنتيكى صفات مطالعه شده در شرايط تنش خشكى و آبيارى نرمال

Table 3. Estimation of genetic parameters of some of agronomic traits in drought stress and normal irrigation conditions

\begin{tabular}{|c|c|c|c|c|c|c|c|c|c|c|c|c|}
\hline \multicolumn{6}{|c|}{ تنش خشكى } & \multicolumn{6}{|c|}{ آبيارى نرمال } & \multirow{3}{*}{ مارارامترهاى زنتيكى } \\
\hline \multirow{2}{*}{ ي بيشرفت زنتيكى } & \multirow{2}{*}{ ترنييرات } & \multirow{2}{*}{ فنغييرات } & \multirow{2}{*}{ وراثتيذيرى } & \multirow{2}{*}{ فواريانس } & \multirow{2}{*}{ واريانس زنتيكى } & \multirow{2}{*}{ ييشرفت رزنتيكى } & \multirow{2}{*}{ ضريب تنغييرات } & \multirow{2}{*}{ ضريب تنييرات } & \multirow{2}{*}{ وراثتيذيرى } & \multirow{2}{*}{ واريانس فنوتيبى } & \multirow{2}{*}{ واريانس رُنتيكى } & \\
\hline & & & & & & & & & & & & \\
\hline$\mu F / \Delta \Delta$ & $18 / \lambda \mu$ & $19 / \mathrm{M}$ & $99 / \%$ & $\Delta \cdot \Delta / \Delta T$ & $\Delta \cdot T / / T$ & $r \mathrm{r} / .9$ & $\mathrm{~W} / / \cdot \mathrm{V}$ & $W / 1 Q$ & $99 / 14$ & $n \cdot / 90$ & $V \cdot F / \Delta V$ & ارتفاع بوته \\
\hline rN/9) & $19 / T V$ & $19 / 99$ & १९/. & $\Delta E / \varepsilon q$ & $\Delta V / \mathrm{V} V$ & $F / / \Delta \Lambda$ & $r \cdot / \Delta$. & $r \cdot|\Lambda|$ & १ร/१४ & $A T / N F$ & $\mathrm{Vq} / 9 \mathrm{~g}$ & طول ميانكره \\
\hline$F q / \Delta T$ & $r \varphi / 9 q$ & $r \omega / \Psi \varphi$ & $q \uparrow / v q$ & $\Delta F / q$. & $\Delta T / \cdot F$ & rN/r. & $19 / \pi \Delta$ & 19/9 & $q \% / \Upsilon q$ & 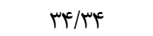 & r & طول برك يرجمه \\
\hline (4) & 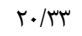 & $r .191$ & $q \vee / r q$ &.$/ .0$ & $\cdot 1 \cdot+1$ & (4) & IV/ar & W/TE & QG/FT & $\cdot / \cdot r$ & . & عرض برى يرجما \\
\hline $91 / 98$ & r/.9 & $r r / \cdot V$ & $9 \Psi / \wedge)$ & $r r / q V$ & $r \cdot / q \mu$ & $\Delta N / I F$ & $r q / v e$ & 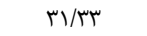 & $9.1 \cdot v$ & $r \mu / T F$ & $r \cdot / \Lambda r$ & تعداد ينجها \\
\hline TV/TE & $\mid r / \Delta \alpha$ & $1 \% / 94$ & $9 \% / 91$ & $15 / 98$ & $10 / \pi$. & 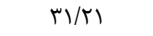 & $|Q / 4|$ & $10 / 99$ & 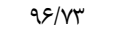 & $19 / \Delta$. & W/AV & طول خوشه \\
\hline$Q N / \Delta \Delta$ & $0.1 . \mu$ & $\Delta T / T_{T}$ & $91 / 44$ & $\mid r / \cdot V$ & $11 / \cdot r$ & $19 V / . r$ & $11 Q / V T$ & $1 f \cdot / . r$ & $9 N / \mu$. & $189 / 99$ & ITT/QT & طول خروج خوشه از غلاف \\
\hline$\Lambda T / M$ & $F 1 / q^{2}$ & FT/VD & $9 \% / 1$. & 1.191 .9 & $9 \Delta 8 / 19$ & $r V / r r s$ & $19 / 41$ & $r \cdot / 9 \pi$ & $\Lambda \& / 9$. & DFA/ $/$ V & FVT/QT & تعداد دانه بِ \\
\hline$|V G /|$. & NV/qT & $9 . / 4$ & $9 \% / \Delta{ }^{2}$ & $V T r / \% \Delta$ & sNT/Vq & $r \cdot \Delta / \wedge \Delta$ & $1 \cdot 1 / \lambda \mu$ & $1 . \mu / V V$ & $q \varepsilon / 4 q$ & TrF/l. & rIQ/QT & تعداد دانه يوك \\
\hline $91 / 4$ & $r .1 \%$ & rl/le & $90 / V T$ & $\Delta \cdots / T r$ & FVA/AT & $r M / T)$ & $11 / \Delta$ & $11 / V^{F}$ & $9 \Delta / 9 \mathrm{~F}$ & $1.1 / 99$ & $1 . F / r \Delta$ & بارورى دانه \\
\hline IS/AF & $N / \mu \cdot$ & N/Fr & QV/.T & $\cdot / v$ & $\cdot / 9 \Lambda$ & תוז/זr & $|r / 9|$ & $|\varepsilon / \mu|$ & $99 / \Delta \Lambda$ & $r / 9 r$ & $1 / A r$ & طول دانه \\
\hline$r q / / f$ & $1 \% / r$ & $\mid F / \notin \Delta$ & १४/৭. &.$/ 14$ & $\cdot 11 \cdot 0$ & $r / \Delta$. & $1 . / 91$ & $1 . / v 9$ & $q \& / \mathrm{V}$ & $\cdot / \cdot v$ & .1 .91 & عرض دانه \\
\hline re/va & $\mid r / T 4$ & $|r / \Delta|$ & $98 / 1 \Delta$ &.$/ \mu$ & $\cdot / r q$ & $r F / \Delta q$ & $1 F / F F^{4}$ & $\mid V / F V$ & $9 N / Y 4$ & $\cdot / 4 \wedge$ & ז & نسبت طول به عرض دانه \\
\hline $99 / 14$ & FN/qT & Fq/א & QN/.r & N.gYAT & $V q \cdot \Delta V V$ & $T V / T \pi$ & $1 \pi / 9$. & $15 / 91$ & $9 F / \Delta F$ & татуя. & rVIrfe. & عملكرد دانه \\
\hline
\end{tabular}


جدول F- همبستىى برخى از صفات زراعى در شر ايط آبيارى نرمال (بالاى قطر) و تنش خشكى (بايين قطر) Table 4. Correlation of some of agronomic traits in normal irrigation (above) and drought stress condition (below)

\begin{tabular}{|c|c|c|c|c|c|c|c|c|c|c|c|c|c|c|}
\hline عملكرد & نبه عرضت طول & عرض دانه & طول دانه & بارورى دانه & تعداد دانه & تعداد دانه & طول خروج خوشه از غلاف & طول خوشه & تعداد ينجه & عرض بركى يرحم & طول برى & طول ميانكره & ارتفاع & صفات \\
\hline r &.$- / 1$ &.$/ . r$ &.$- / \cdot 1$ & $\cdot / \Delta \mathrm{V}^{* *}$ &.$- / 9 Y^{* *}$ &.$- / \cdot \Delta$ &.$- / . r$ & g &.$/ . . F$ &.$- / V Y^{* *}$ & זו/.- &.$/ 4 \Lambda^{*}$ & 1 & ارتفاع بوته \\
\hline$-\cdot / r F$ & .11 & $-\cdot / T \Lambda$ & $-* / 1$ & .1 .9 & $-\cdot 1 \cdot 1$ & - & $.1 \cdot 1$ &.$/ F T^{4}$ &.$/ 4 T$ &.$- / \cdot v$ &.$- / . r$ & 1 & $.1 \Delta 9^{* *}$ & طول ميانكره \\
\hline$-\cdot|r|$ &.$/ 19$ &.$- / \mathbb{e r}^{*}$ &.$- / 19$ &.$- / \% q$ & 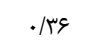 & - - & $-.1 . \mu$ & $\cdot / \mu r$ & $-\cdot 1 \cdot 1$ & .148 & 1 &.$/ 44$ &.$/ 99^{* *}$ & طول برك يرجم \\
\hline-.1 .9 & .1 .9 & - &.$- / 10$ & $-\cdot / \Lambda e^{* *}$ & $\cdot / N^{* *}$ &.$- / 1 V$ & $-. \cdot / \cdot v$ &.$- / 10$ &.$- / 4$. & 1 &.$- / 11$ &.$- / 44$ & 每 & عرض برق يرجم \\
\hline Tr/ &.$/ 1 F$ & .1 .99 &.$/ 19$ & 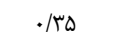 & $-\cdot|\mu|$ & $\cdot / r$. & $-\cdot|r|$ & 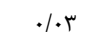 & 1 & $\cdot / r \Lambda$ & $-\cdot 1 \cdot 1$ &.$- / 19$ &.$- / / V$ & تعداد ينجه \\
\hline$-\cdot / r$. &.$/ 19$ & و & -.1 .9 &.$- \cdot 1 \cdot 1$ & 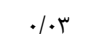 & $-\cdot|r|$ & .1 .9 & 1 &.$- / / 1$ &.$- / 4$. &.$/ 4$ & $\cdot / v \omega^{* *}$ & $\cdot 10 *^{*}$ & طول خوشه \\
\hline$\cdot / r$. &.$- / 1 T$ & $.1 / 9$ & 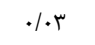 &.$- / . r$ &.- .1 .9 & $-\cdot / r v$ & 1 &.$/ \mathrm{V}$ &.$- / T r$ &.$- / 10$ &.$/ 10$ & .1 .4 & $\cdot \pi$. & طول خروج خوشه از غلاف \\
\hline.$- .1 \cdot 1$ & $-.1 . r$ & r & $\cdot 1 \cdot 1$ & . & -.1 .9 & 1 & $-\cdot / r$ & 每/ & $\cdot / T r$ & -.1 .0 &.$- / 1 T$ & $\cdot / 4 q$ &.$/ \mathrm{V}$ & تعداد دانه پٍر \\
\hline$-\cdot / \Gamma \Lambda$ & . & $-\cdot|\Delta|^{*}$ &.$- / 1 T$ &.$- / 94^{* *}$ & 1 & ع (ז/.• & . &.$/ 14$ & - &.$/ 49^{*}$ &.$/ 4 V$ & .110 & $\cdot / \cdot r$ & تعداد دانه يوك \\
\hline$\cdot / r \Lambda$ &.$- / 49$ & $-\cdot /\left.\Delta\right|^{*}$ &.$/ 1$ & 1 &.$- / 9 Y^{* *}$ &. $\mid 8 \Lambda^{* *}$ & $-\cdot / \mu 1$ & -.1 .1 & 年/ &.$- / 49$ & $-\cdot / T V$ &.$- / .1$ & .1 .4 & بارورى دانه \\
\hline .1 .9 & $\cdot / r^{* *}$ & $\cdot / T V$ & 1 & $\cdot / 4 \Lambda^{*}$ & $-\cdot 109^{*}$ & .111 &.$- / \Delta s^{* *}$ &.$/ . r$ & זו/. &.$- / T q$ & .11 & $-.1 \cdot \Delta$ & $-.1 \cdot 0$ & طول دانه \\
\hline$\cdot / \Delta r^{* *}$ & $-\cdot / \Gamma \wedge$ & 1 & עז/. & $.19 \omega^{* *}$ & $-\cdot / \mathrm{r}^{* *}$ &.$/ \mathrm{V}$ & -.1 .4 &.$- / 1 f$ &.$- / 11$ &.$- / T \Lambda$ & . & $-\cdot / \pi \Delta$ & $-\cdot 11$ & عرض دانه \\
\hline 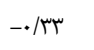 & 1 & $-\cdot / \Lambda r^{* *}$ & .198 &.$- / 4 t$ & $-\cdot / \mathcal{*} \Lambda^{* *}$ &.$/ 1 \Lambda$ & .1 .4 & ( & $-.1 \cdot 1$ & .1 .4 &.$- / 1 \Lambda$ & $-\cdot|\omega|^{*}$ &.$- / 19$ & نسبت طول به عرض دانه \\
\hline 1 &.$- / \Delta q^{* *}$ & $\cdot / N r^{* *}$ &.$\mu$. &.$/ F \Delta$ &.$- / 4 q^{*}$ &.$/ 11$ &.$/ . r$ & 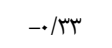 &.$- \cdot 1 \cdot 1$ & $1 \cdot 4$ & $-\cdot / 1 \Lambda$ & $-\cdot \mid \Delta f^{* *}$ &.$- / 19$ & عملكرد دانه \\
\hline
\end{tabular}


عليت و تفسير نتايج حاصل از آن، اطلاعات دقيـقتـر و قابـل

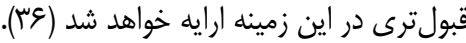

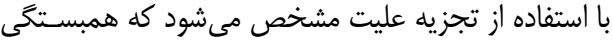

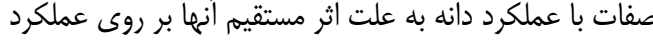

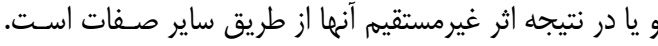

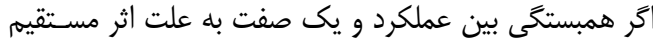

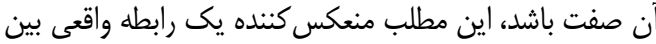

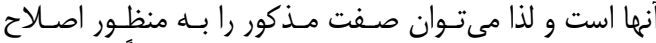

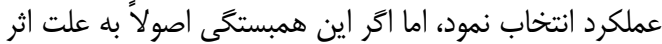

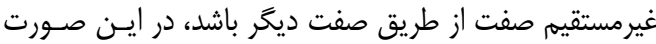

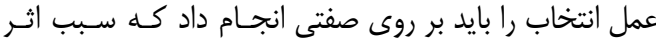

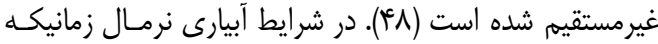

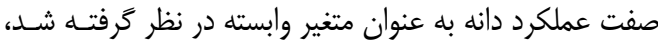

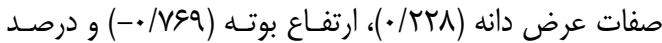

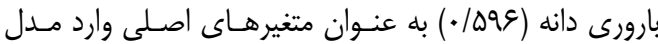

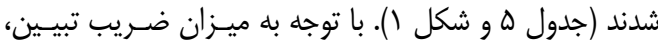

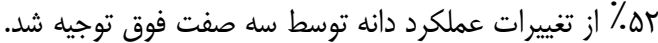

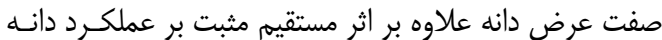

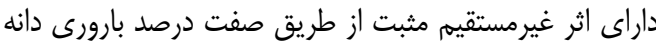

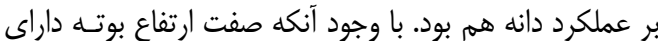

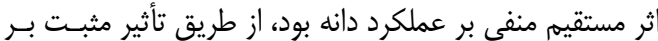

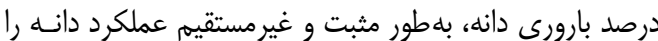

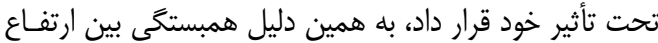

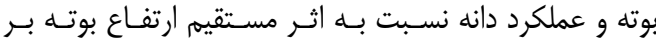

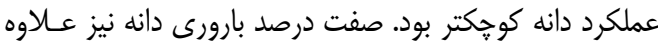

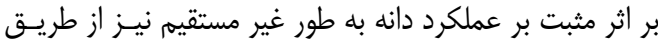

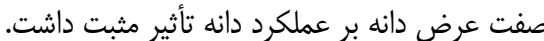

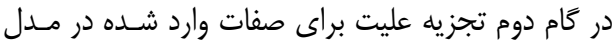

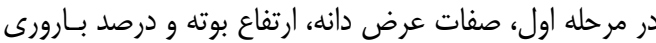

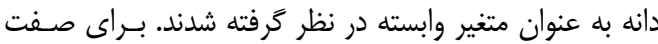

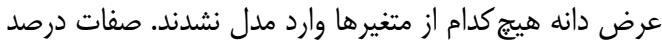

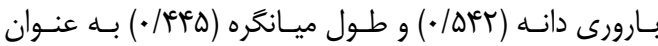

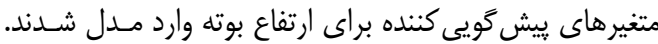

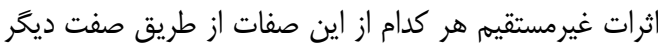

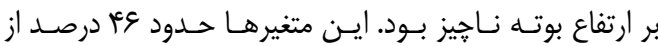

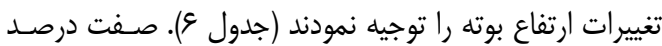

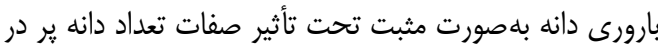

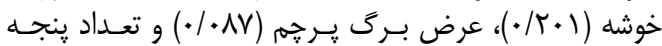

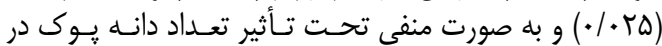

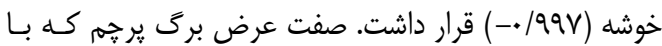

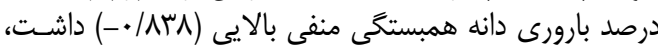

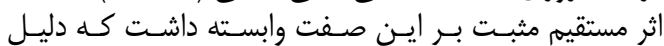

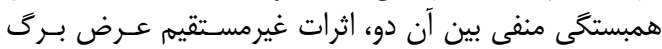

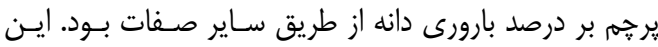

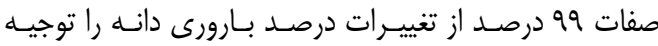

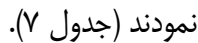

در شرايط تنش خشكى عملكرد دانه با صفات ارتفاع بوتـهـ

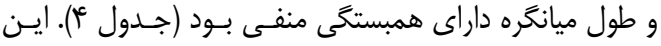

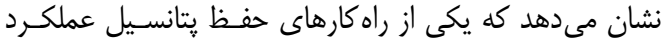

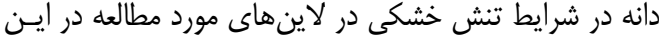

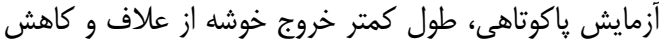

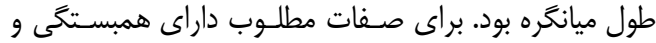

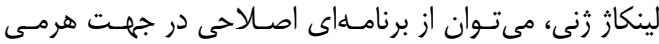

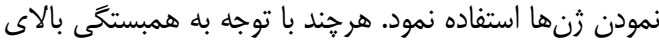

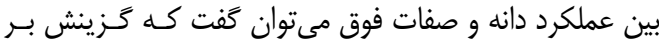

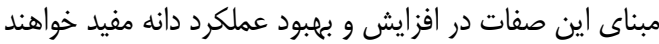

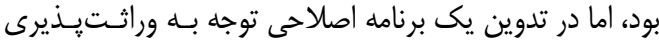

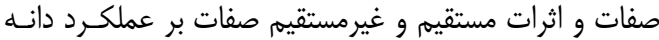

نيز ضرورى است (ع)ات تجزيه ركر سيون و عليت

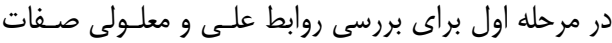

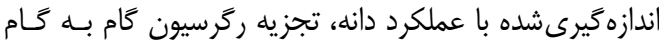

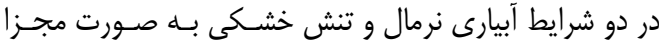

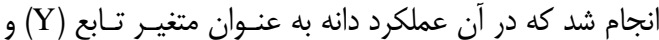

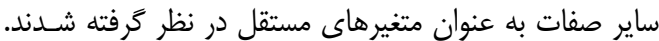

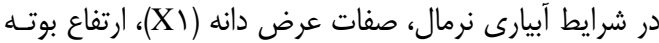

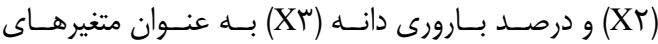

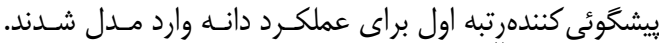

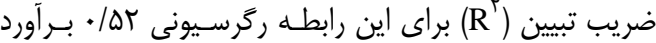

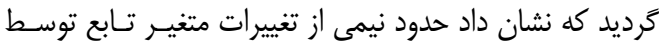

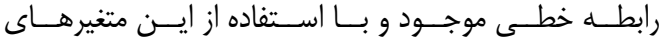

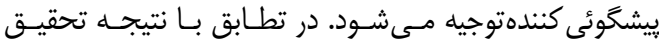

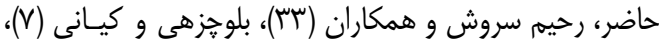

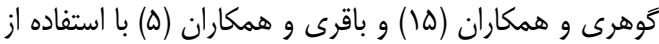

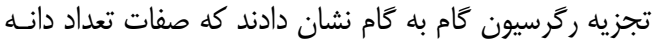

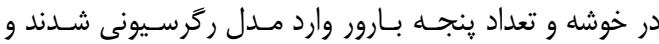

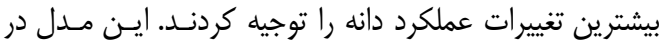

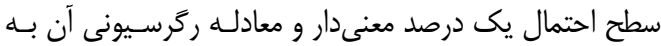

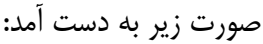
$Y=r q \mu / / r r+\Delta 1 \cdot / T^{\prime} X_{1}-1 V / 99 X_{r}+r \Delta / \Delta q X_{r}$

تجزيه رگرسيون گام به گام در شرايط تنش خشكى نشان

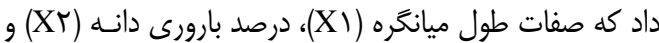

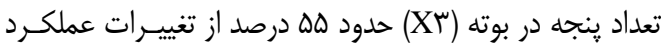
دانه (Y) را توجيه مى نمودند $Y=r q V N / \cdots-V \cdot / r r X_{1}+r r / \Delta r X_{r}-q r / q 1 X_{r}$

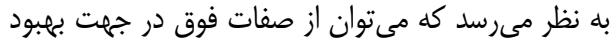

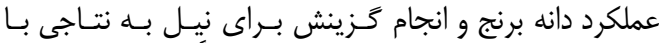

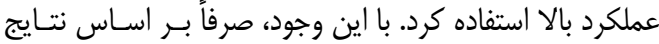

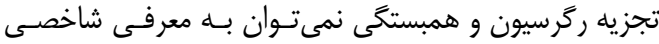

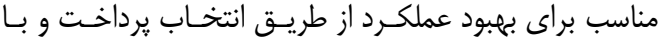

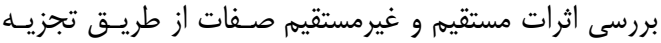



ارزيابى برخى صفات زراعى و ارتباط آنها با عملكرد دانه در لاينهاى موتانت برنج در شرايط آبيارى ..

جدول هـ اثرات مستقيم+ و غير مستقيم متغيرهاى مستقل بر عملكرد دانه به عنوان متغير وابسته در شرايط آبيارى نرمال Table 5. Direct (under lined) and indirect path coefficient on grain yield in normal irrigation condition

\begin{tabular}{|c|c|c|c|c|}
\hline متغير & عرض دانه & ارتفاع بوته & درصد بارورى دانه & اثر كل يا همبستگى با متغير وابسته \\
\hline عرض دانه &.$/ \pi T$ & 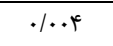 &.$/ / r$ & $\cdot / \Delta F$ \\
\hline ارتفاع بوته & $-.1 \cdot 19$ &.$- / \mathrm{VV}$ &.$- / 44$ & 每/ \\
\hline درصد بارورى دانه & . & $\cdot / \mu F$ & .18 & $\cdot / r \Lambda$ \\
\hline \multicolumn{3}{|c|}{ ضريب تبين (هT/ } & \multicolumn{2}{|c|}{ اثرات باقيمانده ( } \\
\hline
\end{tabular}

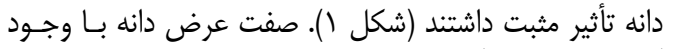

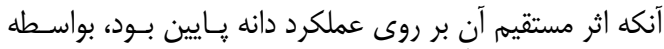

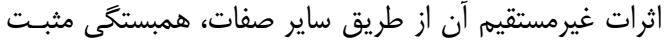
بالايى با عملكرد دانه نشان داد.

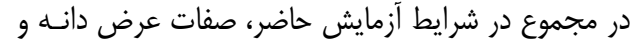

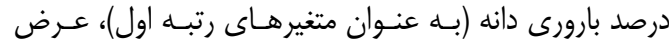

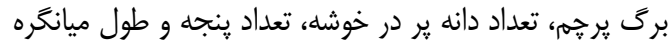

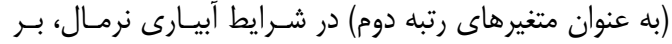
عملكرد

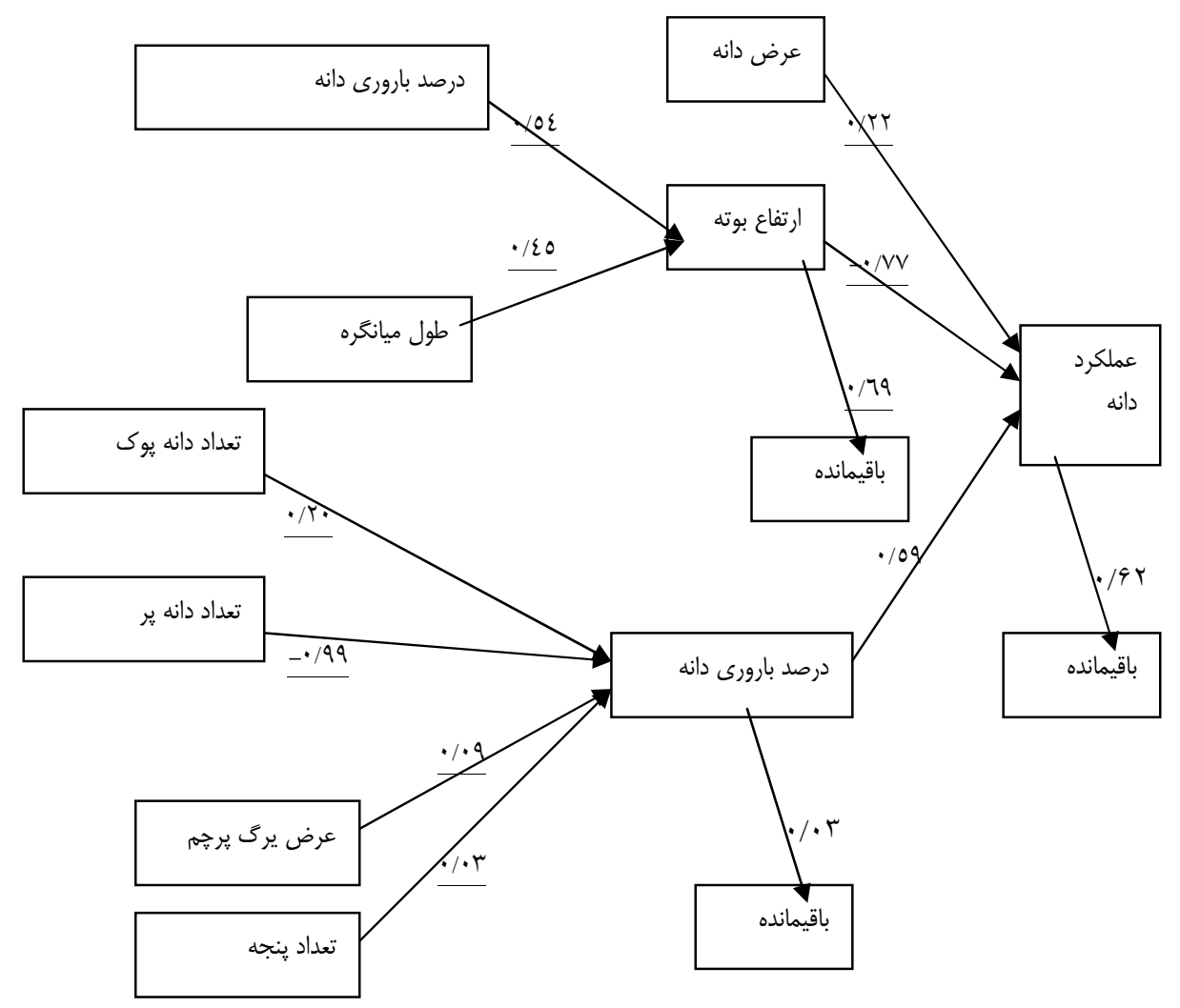

شكل 1- دياگرام ضرايب عليت در شرايط آبيارى نرمال

Figure 1. Path coefficients diagram in normal irrigation condition

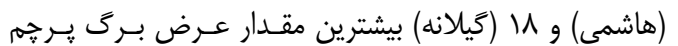

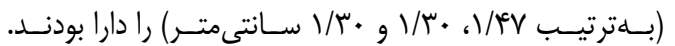

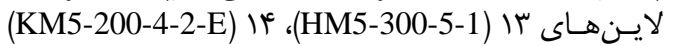

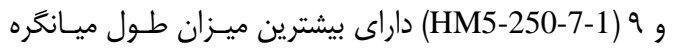

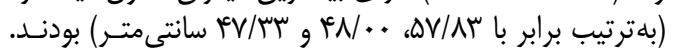

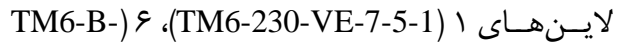

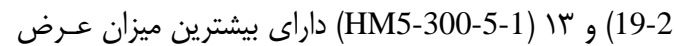

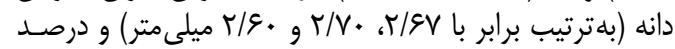

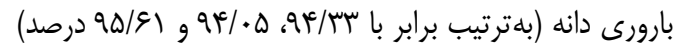

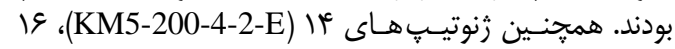




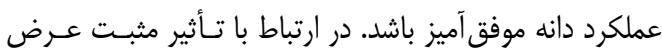

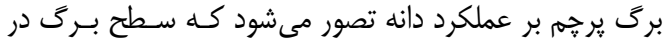

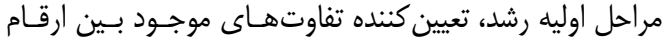

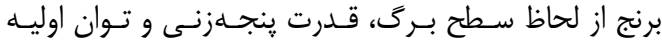

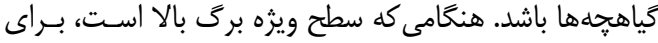

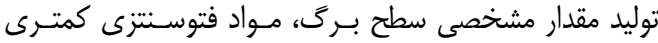

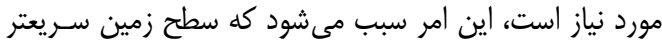

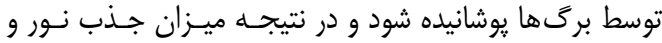

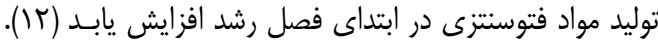

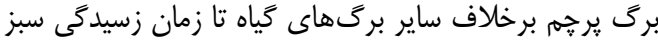

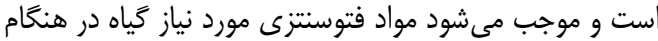

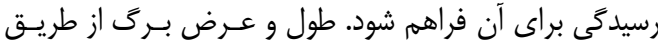

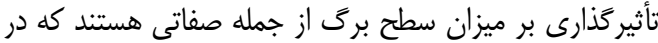

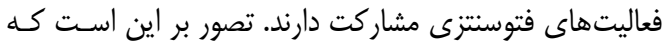

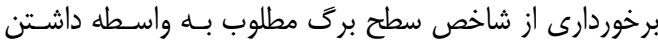

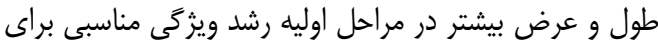

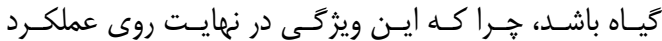

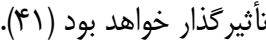

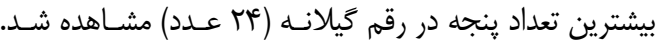

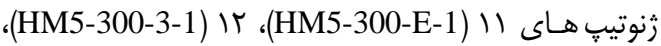

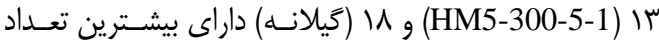

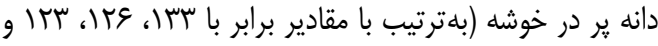

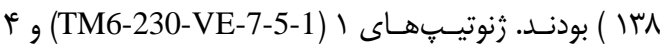

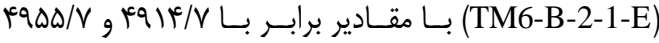
كيلو

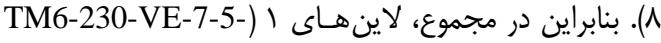

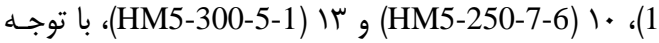

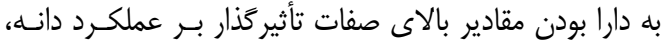

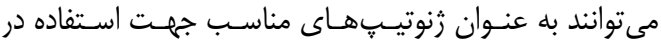

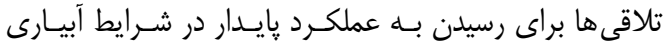

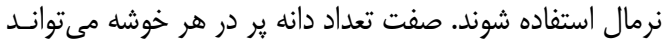

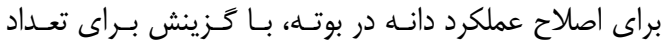

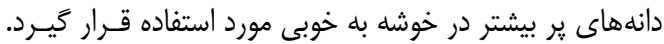

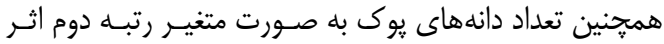

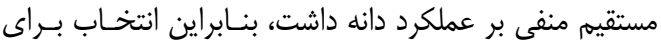

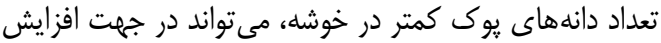

جدول צ- اثرات مستقيم+ و غيرمستقيه متغيرهاى مستقل بر ارتفاع بوته به عنوان متغير وابسته در شرايط آبيارى نرمال Table 6. Direct (under lined) and indirect path coefficient on plant height in normal irrigation condition

\begin{tabular}{|c|c|c|c|}
\hline متغير & درصد بارورى دانه & طول ميانكره & اثر كل يا همبستىى با متغير وابسته \\
\hline درصد بارورى دانه & $\stackrel{. / \Delta F}{ }$ &.$/ \cdot M r$ & $\cdot / \Delta \mathrm{V}$ \\
\hline طول ميانكره & . & $\cdot / F \Delta$ &.$/ 4 \Lambda$ \\
\hline
\end{tabular}

\begin{tabular}{|c|c|}
\hline ضريب تبيين (\&\&/• & اثرات باقيمانده (\&9/. \\
\hline
\end{tabular}

جدول V- اثرات مستقيم+ و غير مستقيم متغيرهاى مستقل بر درصد بارورى دانه به عنوان متغير وابسته در شرايط آبيارى نرمال Table 7. Direct (under lined) and indirect path coefficient on grain productivity in normal irrigation condition

\begin{tabular}{|c|c|c|c|c|c|}
\hline متغير & تعداد دانه بر در & تعداد دانه يوك در & عرض برى يرجم & تعداد ينجه بارور & اثر كلغير وابستهتى با همبتى با \\
\hline تعداد دانه ير در خوشه &.$/ 4$ &.$- / .19$ &.$- / \cdot M F$ & $.1 .4 q$ & $\cdot /$ \\
\hline تعداد دانه يوك در خوشه & .1 .9 &.$- \cdot 99$ & $-\cdot / M$ & $\cdot / r r$ & $-\cdot / 4 t$ \\
\hline عرض برى يرحقم &.$- / \cdot 14$ & $\overline{. / \cdot V^{c}}$ & .1 .9 &.$- / .4$ & $-\cdot / A F$ \\
\hline تعداد ينجه بارور & $\% \circ$ & $-* / \cdot \cdot v$ &.- .1 .1 & 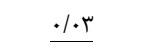 &.$/ \% \Delta$ \\
\hline \multicolumn{3}{|c|}{ ضريب تبين (R/99) } & \multicolumn{3}{|c|}{ اثرات باقيمانده (qr./. } \\
\hline
\end{tabular}

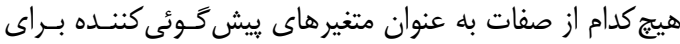

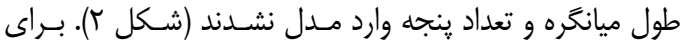

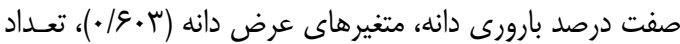

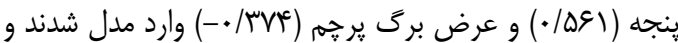

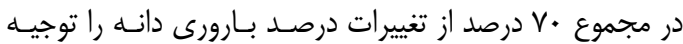

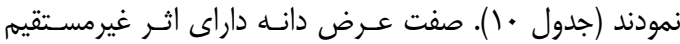

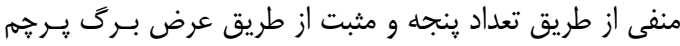

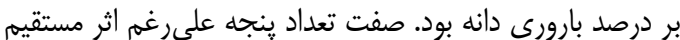

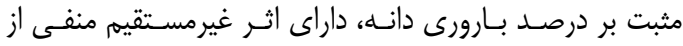

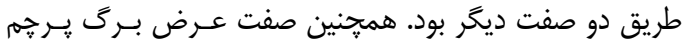

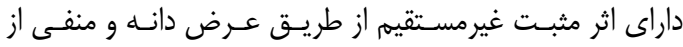

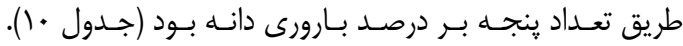

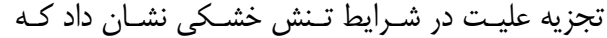

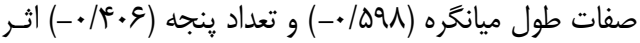

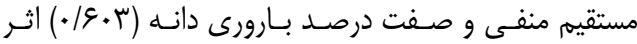

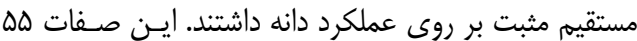

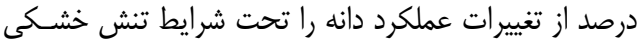

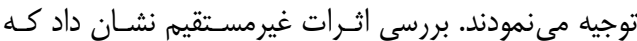

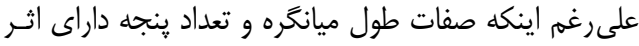

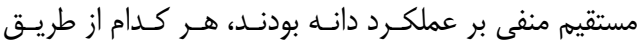

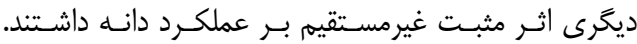

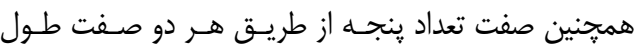

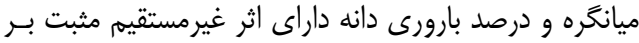

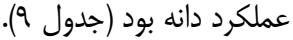

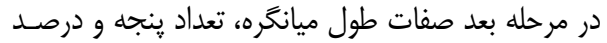
بارورى دانه به عنوان متغيرهاى وابسته در نظر گرفته شدند. 
جدول ^- ميانگين عملكرد دانه و صفات مؤثر بر آن در شرايط آبيارى نرمال Table 8. Mean comparison of grain yield and its components in normal irrigation condition

\begin{tabular}{|c|c|c|c|c|c|c|c|c|c|}
\hline رنوتيب & 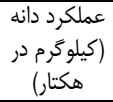 & عرض دانه & (سانتىمتر) & بارورى دانه & طانتىمتر) & 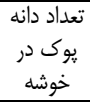 & تعر در خوشه دانه & 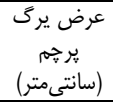 & تعنداد \\
\hline TM6-230-VE-7-5-1 & $F q \mid f / V$ & $r / 9 V$ & $|0| / 9 V$ & 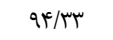 & $F \cdot / Q$. & V & 111 & $1 / 1 T$ & ז \\
\hline TM6-230-VE-8-4-1 & $\vee \Delta \wedge \mathrm{V} /$. & $r / \& V$ & $101 / \cdot \cdot$ & 19/va & rV/Ar & 11 & 94 & $1 / 1$. & r. \\
\hline TM6-250-10-7-1 & FVAF $/$. & $r / q V$ & سK/DF & $19 / 11$ & $F / / V$ & ir & 1.9 & $1 / \cdot v$ & M \\
\hline TM6-B-2-1-E & $\uparrow q \Delta \Delta / V$ & $r / Q V$ & $\mid M F / g V$ & $M / \wedge q$ & rq/^r & ir & 1.1 & $1 / 11$ & 19 \\
\hline TM6-B-7-1 & $F V g \mid / r$ & $r / q V$ & 傮 & $9 / / V)$ & $F r / I V$ & $\wedge$ & $1 . V$ & $1 / N V$ & 19 \\
\hline TM6-B-19-2 & RTEN. & $r / v$. & $\mid \Delta T / . \cdot$ & $9 \% / .0$ & $F \cdot / \Delta$. & v & $11 \pi$ & $1 / .9$ & r. \\
\hline HM5-250-E-1-1 & . & $r / 4$. & سז/FN & $\Lambda Q / T \Delta$ & $\mu \kappa / A \mu$ & 14 & 119 & $1 / \cdot 1$ & if \\
\hline HM5-250-E-3-2 & Feth/T & $r / \mathcal{E V}$ & $\mid Q T / \& V$ & 19/v. & $\Gamma \varphi / \Delta$. & 11 & 98 & $1 / \cdot V$ & 10 \\
\hline HM5-250-7-1 & teted. & $r / \mu$. & $\mid \Delta V / \ldots$ & $9 T / F^{e V}$ & Tr/T & 9 & 111 & $1 / 1$. & 10 \\
\hline HM5-250-7-6 & $1999 / \mathrm{V}$ & $r / \mu$. & $10 \cdot /$. & १)/^। & $F r / I V$ & 1. & 1.9 & $1 / \cdot r$ & r \\
\hline HM5-300-E-1 & $r \cdot V N / r$ & $r / q r$ & $\mid G Y / 9 V$ & 19/Ar & Fis/Ar & 10 & سזו & $1 / \cdot v$ & rI \\
\hline HM5-300-3-1 & $r \Delta V T / r$ & $r / \Delta T$ & $\mid f 9 / 9 V$ & $91 / \% q$ & $F / / \Delta$. & ir & irg &.$/ 9 v$ & r. \\
\hline HM5-300-5-1 & revr/r & $r / \xi$. & $109 / .$. & $90 / 81$ & $\Delta V / A r$ & 9 & שזו & $1 / .9$ & r \\
\hline KM5-200-4-2-E & FTrN/. & سT/r & $\mid r F / .$. & $V w / r f$ & $\mathrm{FN} / .$. & ع & $1 .$. & $1 / q V$ & 10 \\
\hline خزر & $r \cdot r I / r$ & $r / s$. & سب/ & $\Lambda V / \mu$. & $\mid \varphi / .$. & 10 & 1.0 & $1 / 1$. & M \\
\hline ل هاشمى & $f \mid Q V / \mu$ & $r / \mu$. & 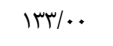 & $V \Delta / \& V$ & $\kappa \varphi / Q$. & ו ו & qv & $1 / r$. & זו \\
\hline 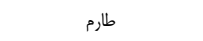 & rఎ৭\&/V & $r / \mu$. & $\mid \& N / 9 V$ & $\Lambda V / \Lambda$. & $F \psi / g V$ & 19 & 118 & $1 / 11$ & $M$ \\
\hline كيلانه & prva/. & $r / q^{*} V$ & $1.4 / 9 V$ & $\Lambda F / T V$ & $r r / g V$ & rq & אזו & $1 / r$. & Tf \\
\hline ميانكين & FEAT/TE & $r / \phi)$ & $\mid f \varepsilon / 91$ & $M / V G$ & $\kappa \Gamma / \Delta Q$ & $\mid f / 4 \pi$ & $111 / 91$ & r/ו & $|v / 9|$ \\
\hline LSD & la &.$/ T$ & $\Delta / \Lambda$. & F/qu & $r / 9 q$ & G/VG & $r \cdot 1 \cdot V$ & .1 .94 & $\Gamma / \Gamma \Delta$ \\
\hline
\end{tabular}

جدول q- اثرات مستقيم+ و غير مستقيم متغيرهاى مستقل بر عملكرد دانه به عنوان متغير وابسته در شرايط تنش خشكى (nم Table 9. Direct (under lined) and indirect path coefficient on grain yield in drought stress condition

\begin{tabular}{|c|c|c|c|c|}
\hline متغير & طول ميانكره & درصد بارورى دانه & تعداد ينجه بارور & اثر كل يا همبستخى با متنير وابسته \\
\hline طول ميانكره &.$- / 4$. & $\cdot / \cdot r r$ &.$/ \cdot 9$ & $-\bullet / \Delta F$ \\
\hline درصد بارورى دانه & $-. / \cdot+4$ & .19. & r & $\cdot / 4 \Delta$ \\
\hline تعداد ينجه بارور & . 1.94 &.$- / 19$ & $-. / 4 \mid$ &.$- / \cdot V V$ \\
\hline
\end{tabular}

$$
\left(R^{2}=/ \Delta ه\right) \text { (اثرات باقيمانده }
$$

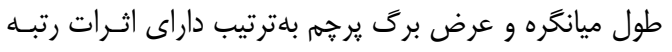

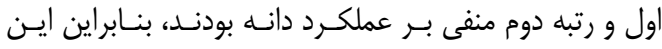

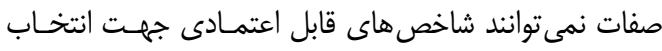

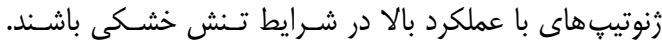

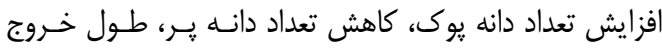

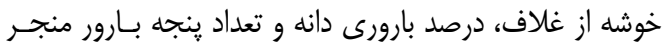

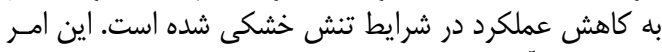

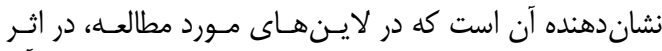

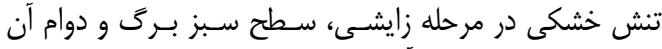

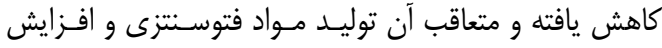

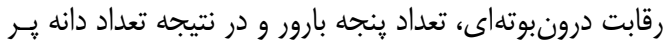

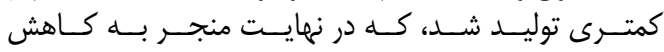

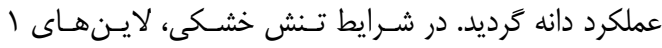

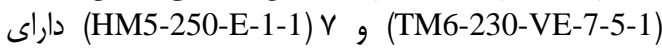

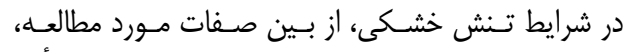

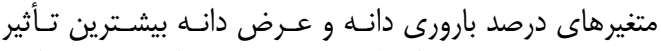

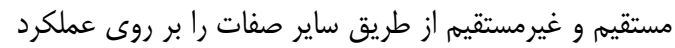

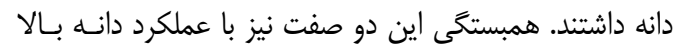

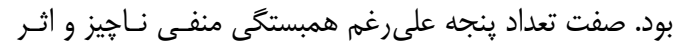

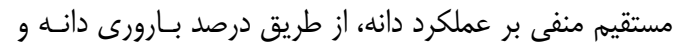

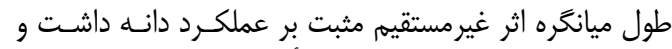

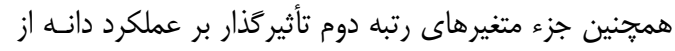

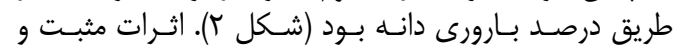

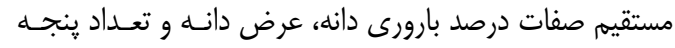

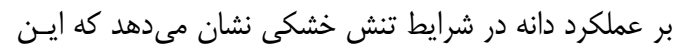

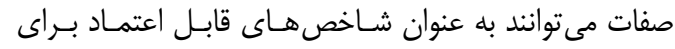

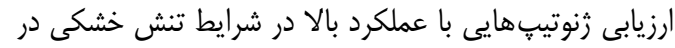

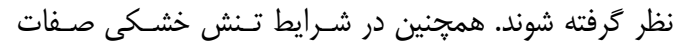




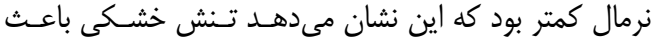

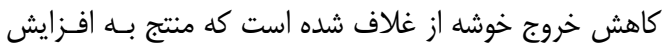

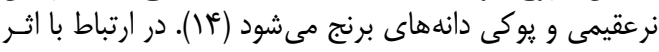

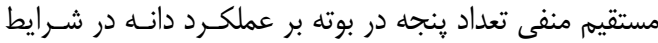

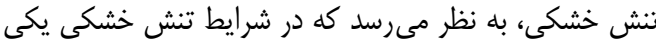

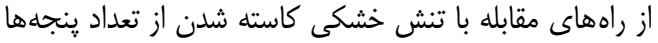

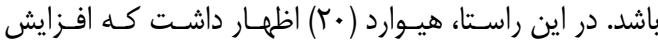

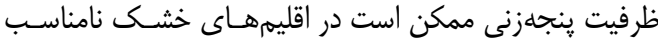

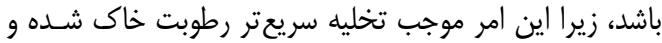

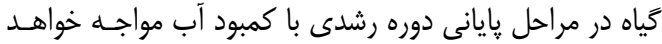

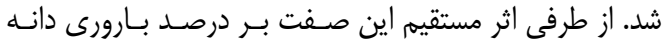

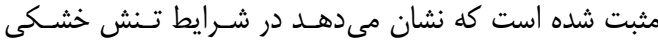

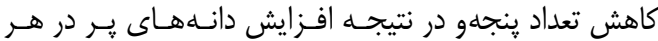

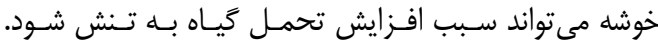

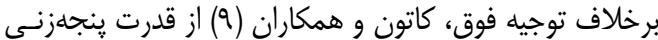

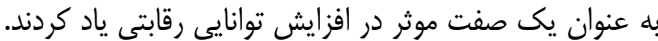

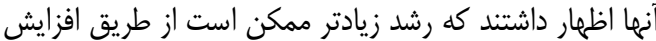

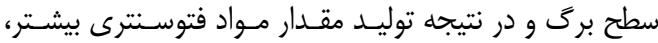

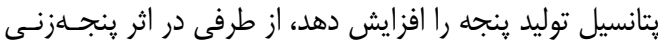

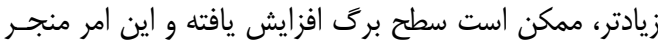

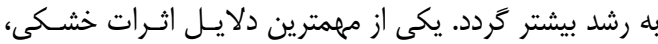

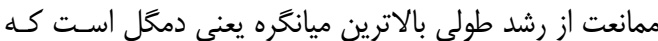

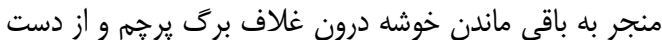

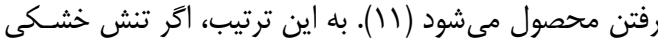

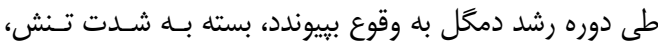

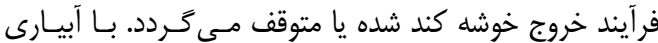

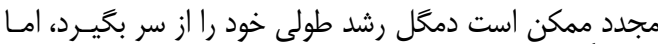

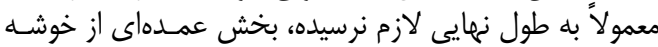
درون غلاف برك يرجم باقى مى ماند.

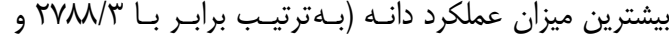
(TVD r (TM6-250-10-7-1) r) (TM6-230-VE-8-4-1) (TM6-B-2-1-E)

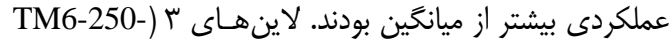

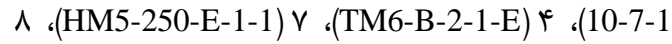
HM5-) (HM5-300-3-1) (HM5-250-E-3-2)

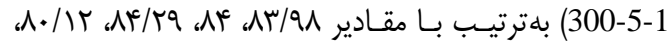

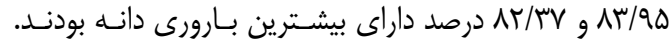

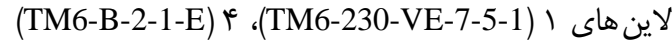

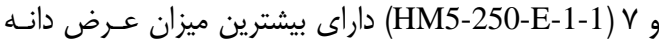

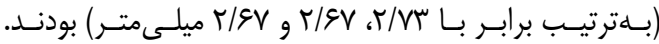

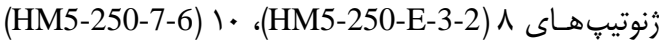

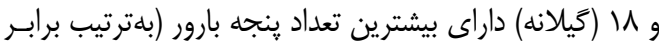

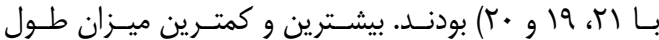

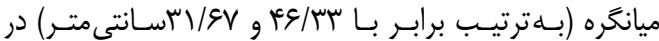

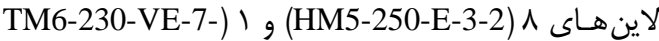

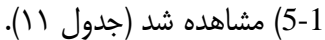

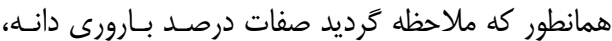

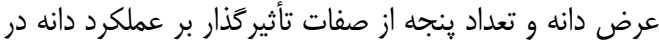

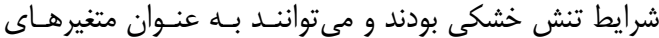

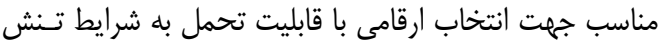

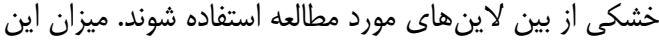

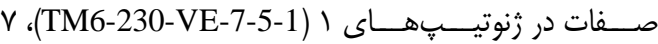
(HM5-300-E-1) I) (HM5-250-E-1-1) (HM5-300-5-1) بيشترين مقادير را نشان مى داد و بنـابراين

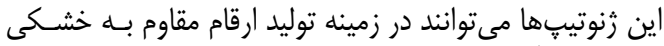

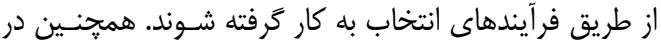
شرايط تنش خشكى، طول خروج خوشه در مقايسه بـا آبيـارى 
19. ارزيابى برخى صفات زراعى و ارتباط آنها با عملكرد دانه در لاينهاى موتانت برنج در شرايط آبيارى

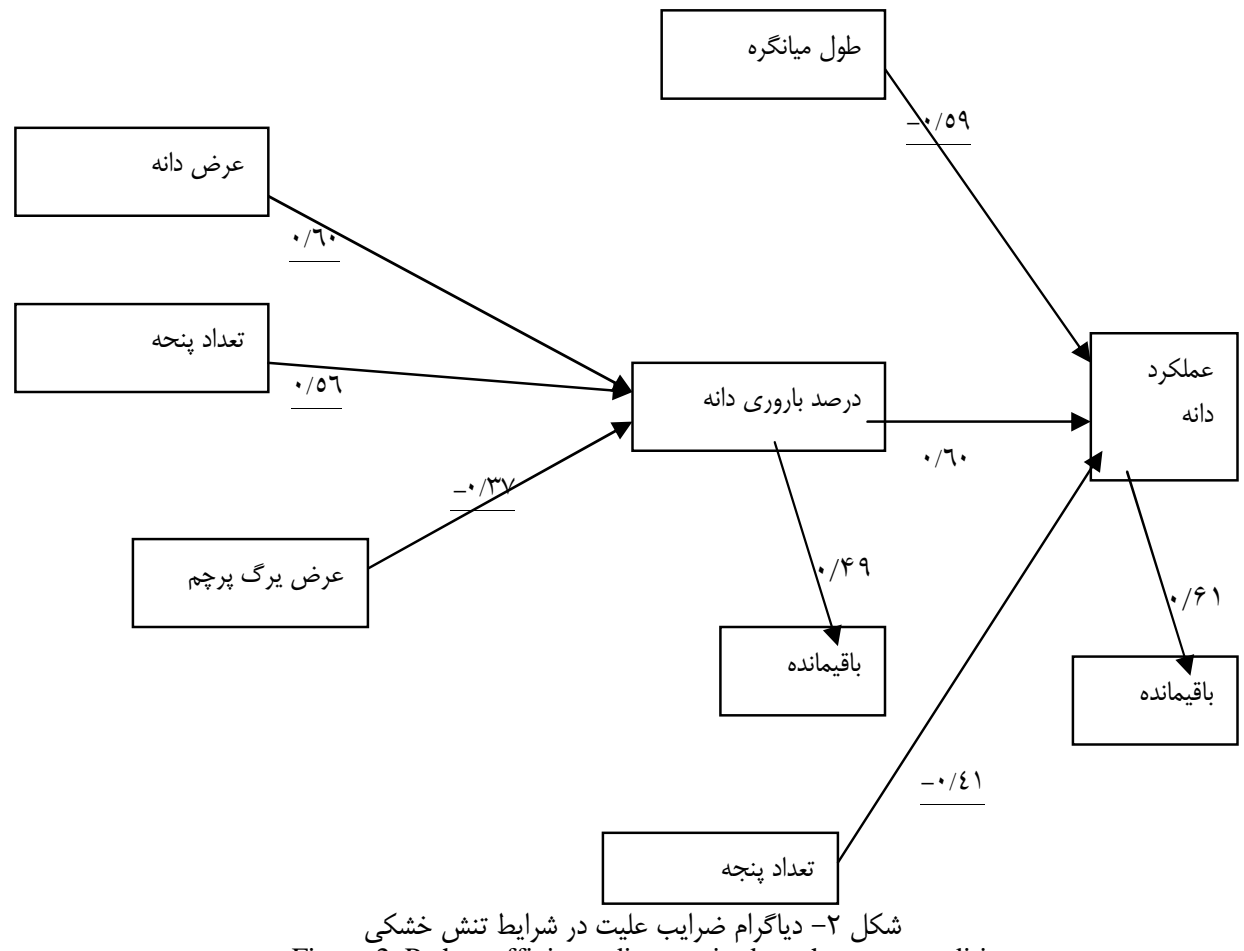

Figure 2. Path coefficients diagram in drough stress condition

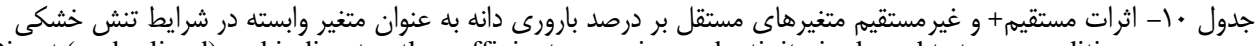
Table 10. Direct (under lined) and indirect path coefficient on grain productivity in drought stress condition

\begin{tabular}{|c|c|c|c|c|}
\hline متغير & عرض دانه & تعداد ينجه بارور & عرض برى يرجم & اثر كل يا همبستكى با متغير وابسته \\
\hline عرض دانه & $\cdot / 9$. & $-.1 .9 \mathrm{~V}$ & $-\cdot / / \Lambda$ & $\cdot / 90$ \\
\hline تعداد ينجه بارور & $-\overline{-.1 .9} \mu$ & 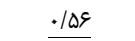 & $\cdot / 19$ & 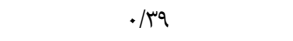 \\
\hline عرض برى يرجهم &.$/ 11$ & $\overline{-. / 11}$ &.$- / r v$ &.$- / r^{\prime}$ \\
\hline \multicolumn{3}{|c|}{ ضريب تبين (R/V) } & \multicolumn{2}{|c|}{ اثرات باقيمانده (qq/•= } \\
\hline
\end{tabular}

جدول 11 - ميانخين عملكرد دانه و صفات مؤثر بر آن در شرايط تنش خشكى

Table 11. Mean comparison of grain yield and its components in drough stress condition

\begin{tabular}{|c|c|c|c|c|c|c|}
\hline رنوتيب & 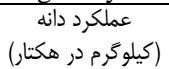 & (ميللىض دانه) & درصد بارورى دانه & 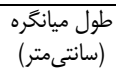 & 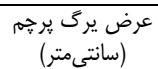 & تعداد ينجه \\
\hline TM6-230-VE-7-5-1 & $r V M N / r$ & $r / V^{N}$ & $9 \% / 94$ & $T I / \& V$ & $1 / 1 r$ & 19 \\
\hline TM6-230-VE-8-4-1 & r|ЯY/. & $r / q \pi$ & $V \backslash / \mp \wedge$ & س & $1 /$. & if \\
\hline TM6-250-10-7-1 & rIVN/T & $T / \mathscr{F V}$ & $\Lambda r / Q \Lambda$ & $r_{\Delta} / .$. & $1 / \cdot$. & iv \\
\hline TM6-B-2-1-E & r|q\%\% & $r / 9 V$ & $\Lambda F /$. & $r \Delta / \bar{Q}\rangle$ & $1 / . r$ & 10 \\
\hline TM6-B-7-1 & $199 \Delta / V$ & $T / V \cdot$ & $\mathrm{W} / \mathrm{s}$. & $r \Delta / .$. & $1 /$. & זו \\
\hline TM6-B-19-2 & $194 /$. & $r / \Delta$. & $V \cdot(\Delta)$ & $f . / 9 V$ & $1 / \mathrm{V}$ & If \\
\hline HM5-250-E-1-1 & TrVQ/. & $r / 9 V$ & $\Lambda F / r q$ & שr/4 & $1 / . r$ & iv \\
\hline HM5-250-E-3-2 & $1998 / \mathrm{V}$ & $T / T^{2}$ & $\lambda \cdot / r$ & س &.$/ 9$. & r) \\
\hline HM5-250-7-1 & $\mid 9 \Lambda \% / r$ & $T / F V$ & $V K / \Delta F$ & $\mu \% /$. &.$/ \mathrm{WV}$ & 19 \\
\hline HM5-250-7-6 & $19 \mathrm{Tr} / \mathrm{V}$ & "r/T & $v g / r \omega$ & Tr/Tr & $1 / \cdot$. & 19 \\
\hline HM5-300-E-1 & $\mid A v V / V$ & $r / T V$ & $\mathrm{~W} / \mathrm{AN}$ & $\mathrm{rN} / .$. &.$/ 9 V$ & 10 \\
\hline HM5-300-3-1 & IVTT/V & $r / \Delta$. & $\Lambda \Gamma / Q \Delta$ & rq/.. & $1 / \cdot \cdot$ & 10 \\
\hline HM5-300-5-1 & $T \cdot T Y /$. & $T / \Delta T$ & $\Lambda T / T V$ & rN/.. & $1 /$. & זו \\
\hline KM5-200-4-2-E & $V Y S / T$ & זו/ץ & $F H / g V$ & rN/.. & r & 1. \\
\hline خزر & $9 \times T / \mathrm{V}$ & $r / \mu$. & $9 \mathrm{~V} / .$. & $F V / G V$ &.$/ 9 v$ & 10 \\
\hline هاشمى - ل ماشى & $19 \% \cdot / V$ & $r / 1$. & FT/gT & rq/.. & $1 /$. & ir \\
\hline طارم & $1 \Lambda \omega q / \Gamma$ & $r / T$. & $G / 4)$ & $F F / .$. & $\mathrm{I} / \mathrm{V}$ & 10 \\
\hline كيلانه & $\mid Q V Q / G V$ & $r / N V$ & $99 / \pi$. & 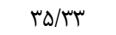 & 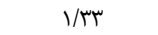 & $r$. \\
\hline ميانكَين & $|\Lambda| \xi / \Lambda \mu$ & 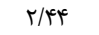 & $V / / \mu r$ & $r q / r f$ & $1 / .4$ & $1 \omega / \pi \omega$ \\
\hline LSD & rqه/qr &.$/ 1 r$ & $1 . / \Lambda \Delta$ & $r / \Delta V$ & $\cdot 1 \cdot 1$ & $\Gamma / \Delta S$ \\
\hline
\end{tabular}


متغير رتبه اول) و شرايط تنش (با توجه به همبسـتخى منفىى)

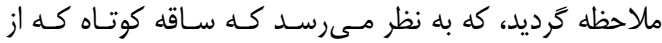

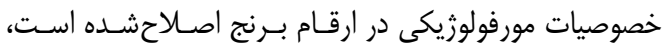

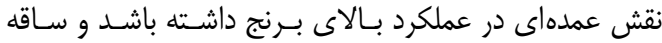

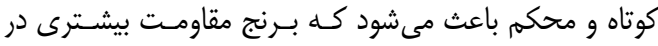

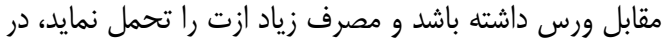

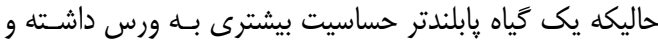

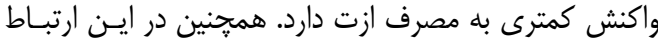

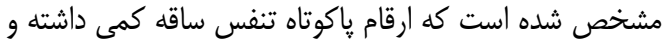

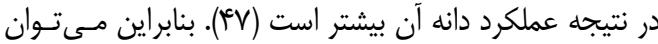

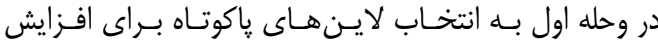

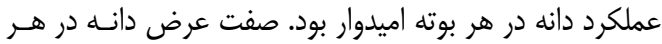

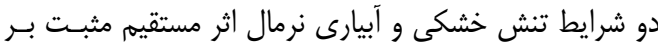

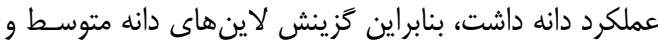

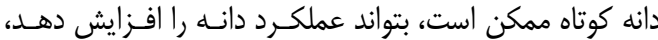

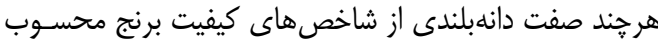

شاخص تنش

اثرات تنش خشكى، بر روى تنى تمام صفات، در قالب

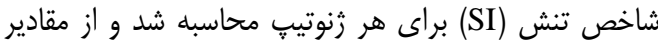

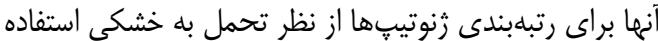

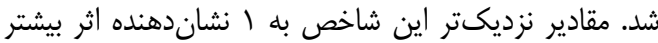

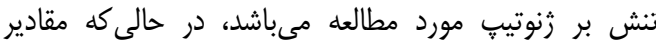

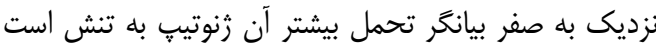

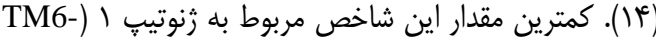

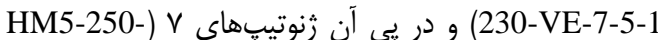
(E-1-1

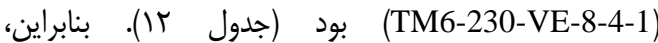

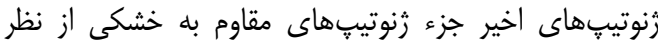

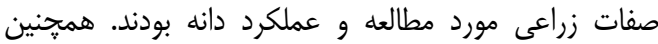

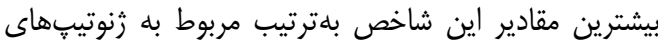

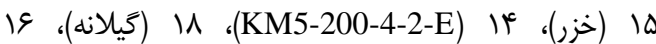

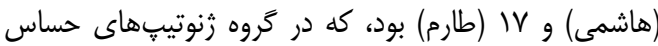

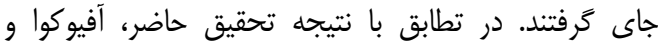

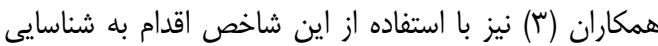

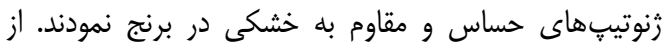

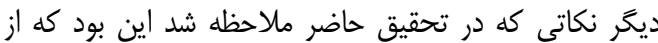

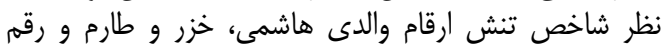

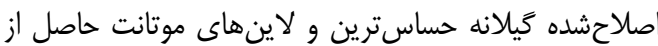

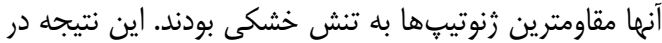

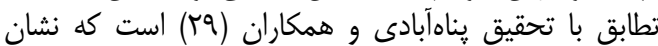

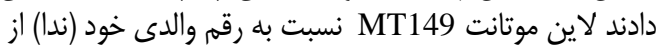

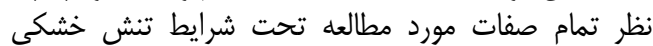

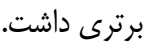

مجموع نتايج تجزيـهـ ركرسـيون و عليـت حـاكى از تـأثير

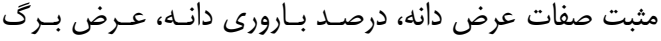

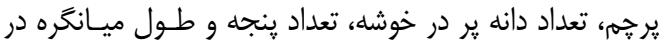

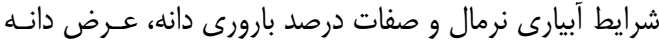

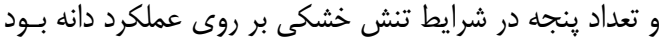

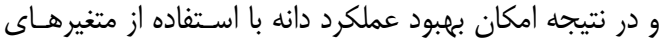

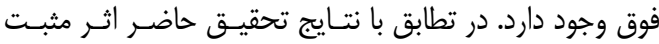

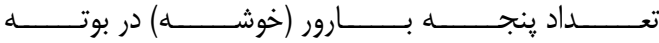

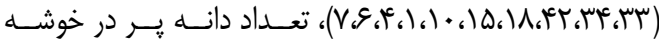

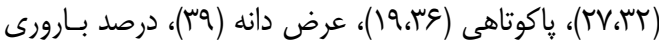

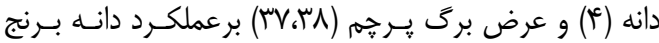

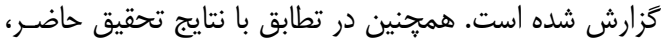

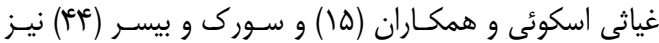

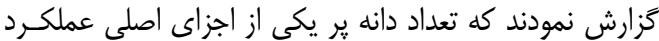

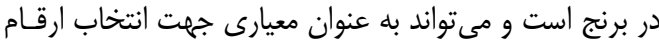

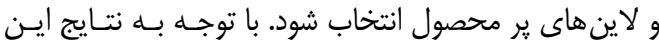

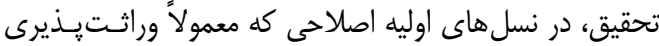

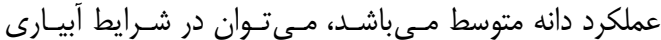

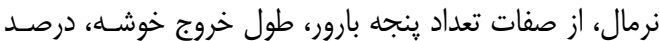

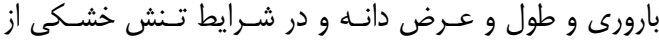

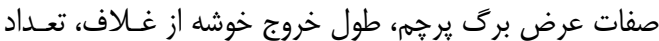

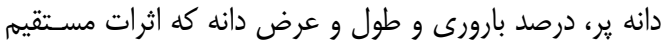

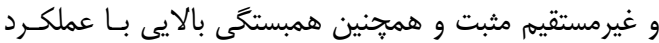

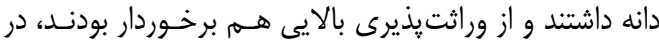

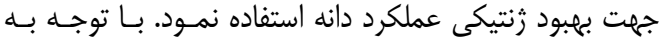

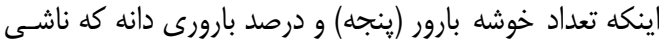

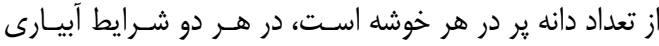

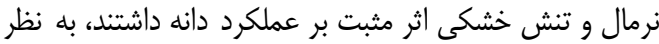

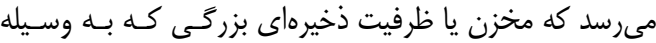

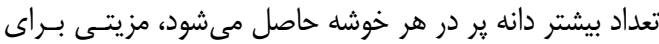

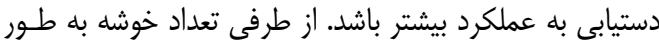

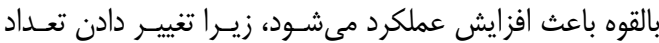

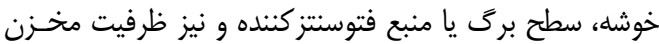

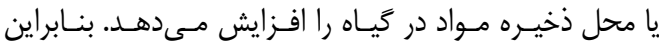

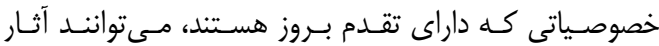

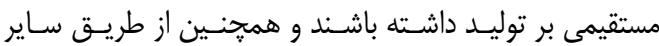

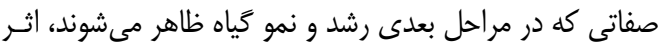

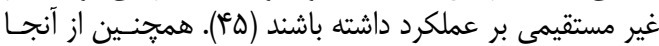

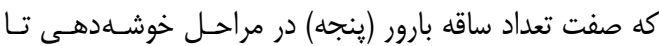

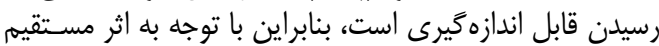

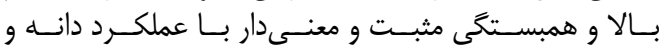

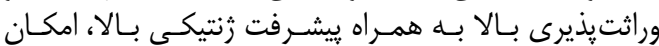

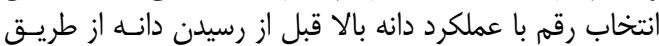

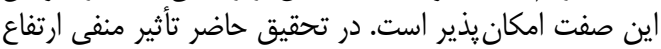

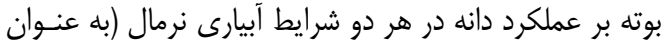




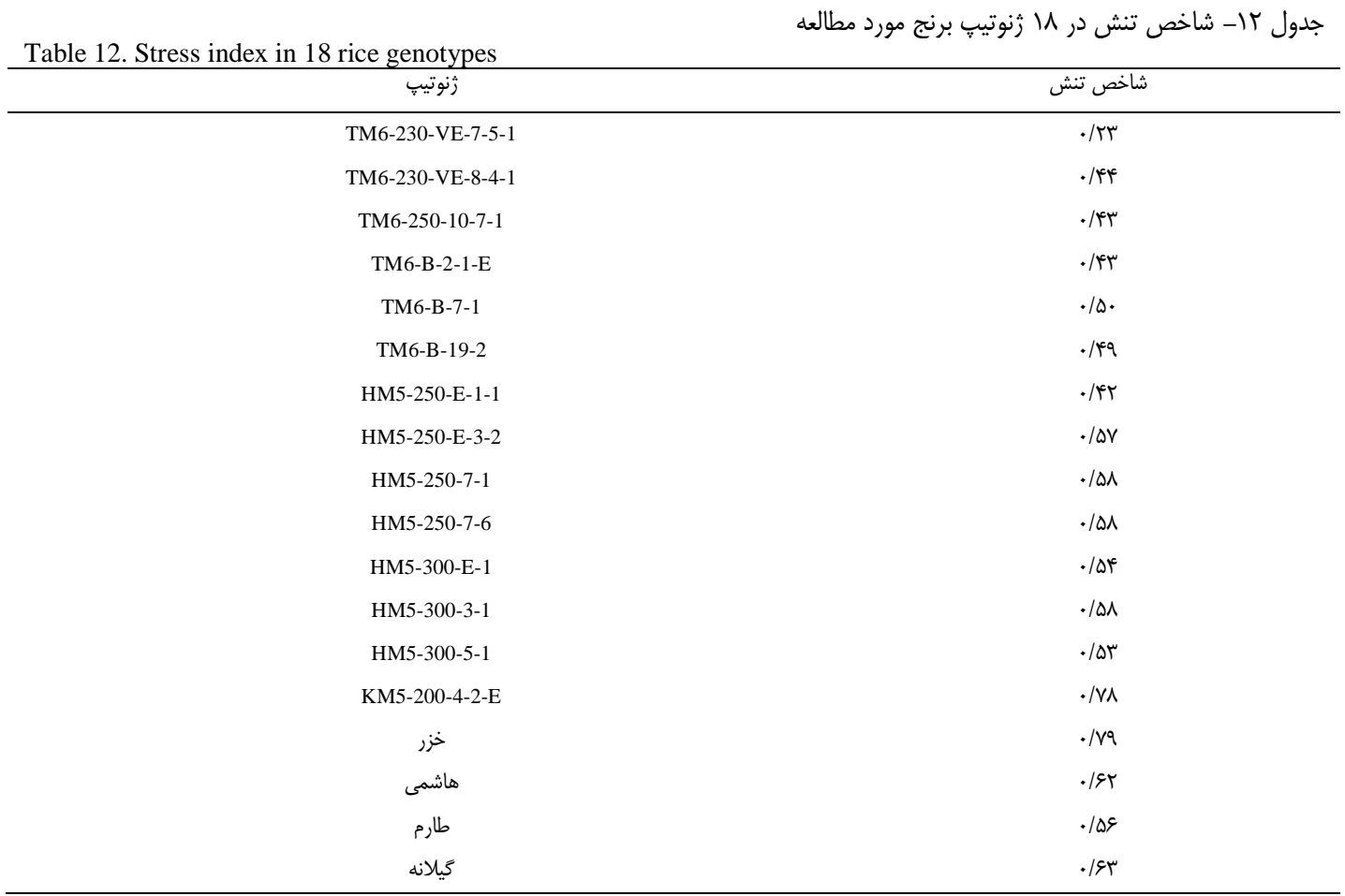

انتخاب به كار كرفته شوند. اين نتايج همجنين نشاند دهنده تأثير

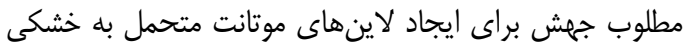

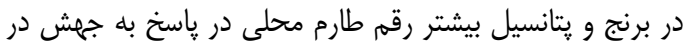

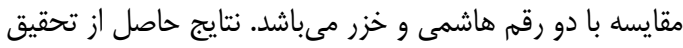

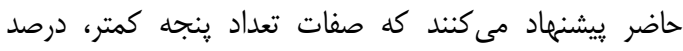

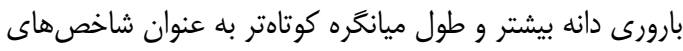

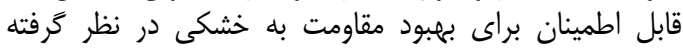

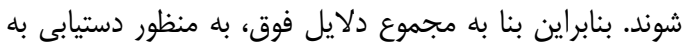

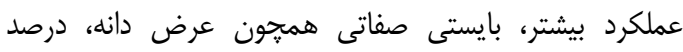

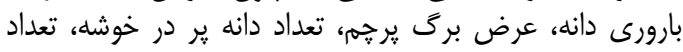

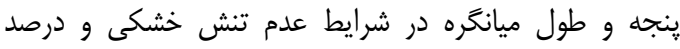

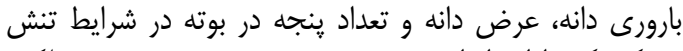

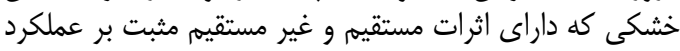

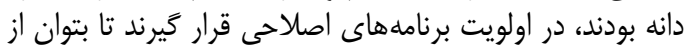
اين صفات در تز ينش زنوتبِ بنهاى با عملكرد بالا استفاده كرد.
در مجموع با توجه به نتايج حاصل از اين تحقيق

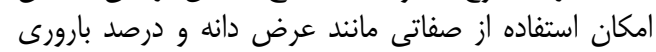

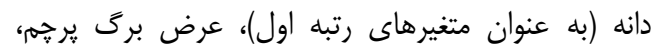

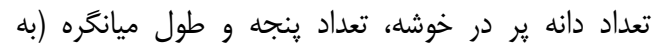

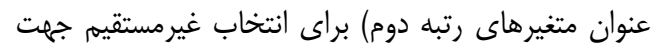

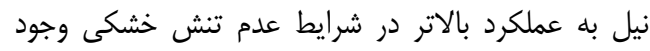

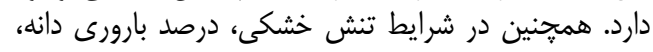

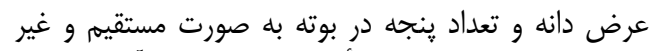

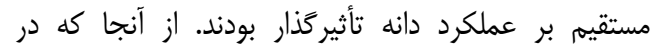

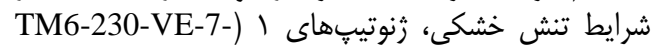

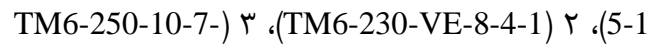

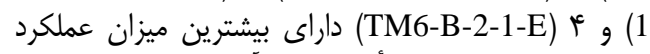

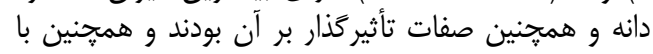

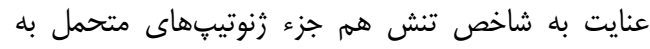

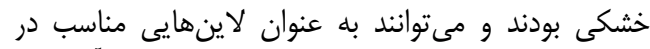
بحث توليد ارقام متحمل به خشكى اندى از طريق فرآيندهاى 
1. Abouzari Gazafrodi, A., R. Honarnejad, M.H. Fotokian and A Alami. 2007. Study of correlations among agronomic traits and path analysis in rice (Oryza sativa L.). Journal of Science and Technologyin Agriculture and Nature Resources, 10(2): 99-106 (In Persian).

2. Adilakshmi, D. and M. Girija Rani. 2012. Variability, character association and path analysis in rice varieties under submergence. Crop Research, 44(1\&2): 146-151.

3. Afiukwa, C.A., J.O. Faluyi, C.J. Atkinson, B.E. Ubi, D.O. Igwe and R.O. Akinwale. 2016. Screening of some rice varieties and landraces cultivated in Nigeria for drought tolerance based on phenotypic traits and their association with SSR polymorphisms. African Journal of Agricultural Research, 11(29): 2599-2615.

4. Aminpanah, H. and P. Sharifi. 2013. Path analysis of rice (Oryza sativa L.) grain yield and its related components in competition with barnyard grass [Echinochloa crus-galli (L.) P. Beauv. Journal of crop Production and Proccessing, 3: 105-121 (In Persian).

5. Bagheri, N.A., N.A. Babaeian-Jelodar and A. Pasha. 2011. Path coefficient analysis for yield and yield components in diverse rice (Oryza sativa L.) genotypes. Biharean Biologist, 5(1): 32-35.

6. Bakar, B.B., N.A.N. Lajili, S. Suhaimi, A. Suzana and J. Abdul Munir. 2005. Path analysis of two sympatric weed species (Echinochloa crus-galli ssp.crus-galli (L.) Beauv. and Ischaemum rugosum Salisb.) in competition with rice (Oryza sativa L. var. MR84) - a comparative study. Plant Protection Quarterly, 20(2): 67-73.

7. Balouchzaehi1 A.B. and G. Kiani. 2013. Determination of selection criteria for yield improvement in rice through path analysis. Journal of Crop Breeding, 5(12): 75-84 (In Persian).

8. Beikzadeh, H., S.M. Alavi Siney, M. Bayat and A.A. Ezady. 2015. Estimation of genetic parameters of effective agronomical traits on yield in some of Iranian rice cultivar. Agronomy Journal (Pajouhesh \& Sazandegi), 104: 73-78 (In Persian).

9. Caton, B.P., A.E. Cope and M. Mortimer. 2003. Growth traits of diverse rice cultivars under severe competition: Implications for screening for competitiveness. Field Crops Research, 83:157-172.

10. Chauby, P.K. and R.P. Singh. 1994. Genetic variability, correlation and path analysis of yield and yield components of rice. Madras Agricultural Journal, 81: 468-470.

11. Dey, M.M. and H.K. Upadhyaya. 1996. Yield loss due to drought, cold and submergence tolerance. Journal of Rice Research in. Asia, 5: 63-66.

12. Dingkuhn, M., D.E. Johnson, A. Sow and A.Y. Audebert. 1999. Relationships between upland rice canopy characteristics and weed competitiveness. Field Crops Research, 61: 79-95.

13. Fischer, R.A. and R. Maurer. 1978. Drought resistance in spring wheat cultivar: I- Grain yield response. Australian Journal of Agriculture Research, 29: 897-912.

14. Ghiasy Oskoee, M., H. Farahbakhsh, H. Sabouri and G. Mohammadinejad. 2012. Effect of drought stress on yield and yield components in rice landraces and improved cultivars under Gonbad Kavous environmental condition. Cereal Research, 2(3): 165-179 (In Persian).

15. Gohari, M., M. Khayat, and S. Lack. 2010. Correlation and path analysis in some of important agronomic traits on grain yield of rice cultivars. New Finding in Agriculture, 4(3): 261-269 (In Persian).

16. Haider, Z., A.S. Khan and S. Zia. 2012. Correlation and path coefficient analysis of yield components in rice (Oryza sativa L.) under simulated drought stress condition. American-Eurasian Journal of Agricultural and Environmental Science, 12(1): 100-104.

17. Haider, Z., A. Razaq, A. Mehboob, S. ur Rehman, A. Iqbal, A. Hussain, U. Saeed, M.T. Naveed, S. $\mathrm{Zia}$, Z. Mahmood and K. Mehmood. 2013. Comparison of associations among yield and yield components in rice (Oryzae sativa L.) under simulated drought stress condition using multivariate statistics. International Journal of Scientific and Engineering Research, 4 (8): 329-340.

18. Hasan-Nataj, E., M. Pouryousef, N. Babaeian-Jelodar, H. Pirdashti and N. Bagheri. 2013. Investigation of morphological traits related to yield of rice (Oryza Sativa L.) promising lines. Journal of Crop Breeding, 5(11): 34-48 (In Persian).

19. Honarnejad, R. 2003. Study of correlation between some quantitative traits and grain yield in rice (Oryza sativa L.) using path analysis. Iranian Journal of Crope Sciences, 1: 25-34 (In Persian).

20. Hurd, E.A. 1971. Can we breed for drought resistance? In: Larson K. L. and Rachter J. D. (Eds). Drought injury and in resistance crop. CSSA Special Publication II, Crop Science Society of America, USA. pp: 77-88.

21. Jayasudha, S. and D. Sharma. 2010. Genetic parameters of variability, correlation and path-coefficient for grain yield and physiological traits in rice (Oryza sativa L.) under shallow lowland situation. Electronic Journal of Plant Breeding, 1(5): 33-38.

22. Jahani, M., G. Nematzadeh and G. Mohammadi Nejad. 2015. Evaluation of Agronomic Traits Associated with Grain Yield in Rice (Oryza sativa) Using Regression and Path Analysis. Journal of Crop Breeding, 7(16): 115-122 (In Persian).

23. Johnson, H.W., H.F. Robinson and R.E. Comstock. 1955. Estimates of genetic and environmental variability in soybean. Agronomy Journal. 47: 314-318.

24. Majd, F. and M.R. Ardakani. 2004. Nuclear Techniques in Agriculture Sciences. Tehran University Press, $381 \mathrm{pp}$.

25. Majidi, T. 2011. Path analysis software. Available in: http://www.pathanalysis.mihanblog.com/.

26. Mohammadi, S.A., B.M. Prasanna and N.N. Singh. 2003. Sequential path model for determining interrelationships among grain yield and related characters in maize. Crop Science, 43: 1690-1697.

27. Mumeni, A., A.A. Zali and P. Vejdani. 1996. Study correlations and path analysis for some of important agronomic traits on rice grain yield. Fourt Iranian Agronomy and Plant Breeding Congress, Esfahan University, 28-30 August, (In Persian). 
28. Nematzadeh, G.A., A.J. Ali, M. Sattari, A. Valizadeh, E. Alinejad and M.Z. Nouri. 2006. Relationship between different allogamic associated trait characteristics of five newly developed cytoplasmic male sterile lines in rice. Journal of Central European Agriculture, 7(1): 49-56.

29. Panahabadi, R., A. Ahmadikhah and H. Askari. 2016. The effects of drought stress on morphophysiological characters and expression of OsCat $A$ in rice seedling. Journal of Agricultural Biotecnology, 8(2): 1-16 (In Persian).

30. Pandey, V.R., P.K. Singh, O.P. Verma and P. Pandey. 2012. Inter-relantionship and path coefficient estimation in rice under salt stress environment. International Journal of Agricultural Research, 1: 116.

31. Pantuwan, G., S. Fukai, M. Cooper, S. Rajatasereekul and J.C. O’Toole. 2002. Yield response of rice (Oryza sativa L.) genotypes to different types of drought under rainfed lowlands. Plant factors contributing to drought resistance. Journal of Field Crops Research, 73: 181-200.

32. Prakash, S. and B.G. Prakash. 1987. Path analysis in ratoon rice. Rice Abstract, 24: 215-218.

33. Rahim Souroush, H., M. Mesbah and A. Hosseinzadeh. 2005. Study relations among yield and yield component traits in rice. Iranian Journal of Agriculture Sciences, 35(4): 983-993 (In Persian).

34. Ravindra Babu, V., K. Shreya, K.S. Dangi, G. Usharani and A.S. Shankar. 2012. Correlation and path analysis studies in popular rice hybrids of India. International Journal of Scientific and Research Publications, 2(3): 1-5.

35. Roy, D. 2000. Plant breeding analysis and exploitation of variation. Alpha Science. 798 pp.

36. Sabori, H., G. Mohammadinejad and M. Fazlalipour. 2011. Selection for improve rice yield by multivariate analysis. Iranian Journal of Field Crops Research, 9(4): 639-650 (In Persian).

37. Sabori, H., A. Rezai, S.A.M. Mirmohammady Maibody and M. Esfahani. 2006. Path analysis for rice grain yield and related traits in tow planting patterns. Journal of Science and Technologyin Agriculture and Nature Resources, 9(1): 113-128 (In Persian).

38. Sarawgi, K.A., N.K. Ratagi and D.K. Soni. 1997. Correlation and path analysis in rice accessions from Madhya Pradesh. Field Crop Science, 38: 1130-1136.

39. Satheeshkumar, P. and K. Saravanan. 2012. Genetic variability, correlation and path analysis in rice (Oryza Sativa L.). International Journal of Current Research, 4: 82-85.

40. Selvaraj, C.I., P. Nagarajan, K. Thiyagarajan, M. Bharathi and R. Rabindran. 2011. Genetic parameters of variability, correlation and path coefficient studies for grain yield and other yield attributes among rice blast disease resistant genotypes of rice (Oryza Sativa L.). African Journal of Biotechnology, 10(17): 3322-3334.

41. Shahid-Masood, M., A. Javaid, M. Ashiq Rabbani and R. Anwar. 2005. Phenotypic diversity and trait association in bread wheat (Triticum aestivum L.) landraces from Baluchistan, Pakistan. Pakistan Journal of Botany, 37: 949-957.

42. Sharifi P., H. Dehghani, A. Mumeni and M. Moghaddam. 2013. Study genetic relations of some of rice agronomic traits with grain yield by some of multivariate analysis. Iranian Journal of Crop Science, 44(1): 169-179 (In Persian).

43. SPSS. 2008. The SPSS System for Windows. Release 22.0. SPSS Inc., IBM Company Headquarters, USA.

44. Surek, H. and N. Beser. 2005. Selection for grain yield and its components in early generations in rice (Oryza sativa L.). Trakya University Journal Sciences, 6: 51-58.

45. Vaezi, S., S. Abde-Mishani, B. Yazdi-Samadi and M.R. Ghanadha. 2000. Correlation and path analysis in maize yield and yield components. Iranian Journal of Agriculture Science, 31(2): 71-82 (In Persian).

46. Venuprasad. R., H.R. Lafitte and G.N. Atlin. 2007. Response to direct selection for grain yield under drought stress in rice. Crop Science, 47: 285-293.

47. Watson, P.R., D.A. Derksen, R.C. Van Acker and M.C. Blrvine. 2002. The contribution of seed, seedling and mature plant traits to barley cultivar competitiveness aginest weeds. Proc. National Meeting- Canadian Weed Science Society, pp: 49-57.

48. Yadav, R.B., R.K. Dubey, M.K. Srivastava and K.K. Sharma. 1995. Path coefficient analysis under three densities in rice. Journal of Soils and Crops, 5(1): 43-45. 


\title{
Evaluation of some Agronomic Traits and their Association with Grain Yield in Mutant Rice Lines under Normal and Post-anthesis Drought Stress Conditions
}

\section{Peyman Sharifi $^{1}$, Ali Akbar Ebadi ${ }^{2}$ and Hashem Aminpanah ${ }^{3}$}

1- Associate Professor, Department of Agronomy and Plant Breeding, Rasht Branch, Islamic Azad University, Rasht, Iran (Corresponding author: peyman.sharifi@gmail.com)

2- Assistant Professor and Member of the Scientific Council of Rice Researches of the Country, Agricultural

Research, Education and Promotion Organization, Rasht, Iran

3- Associate Professor, Department of Agronomy and Plant Breeding, Rasht Branch, Islamic Azad University, Rasht, Iran

Received: January 15, 2017

Accepted: January 13, 2018

\begin{abstract}
In order to evaluate the effect of drought stress and determine of effective traits on grain yield and estimate of genetic parameters, 18 mutant rice lines were studied in normal and drought stress conditions. The experiments were carried out separately using randomized complete block design with three replications during cropping season 2014-2015 at Iranian Rice Research Centers in North of Iran, Rasht. High values of heritability, genetic advances and genetic variances were observed for plant height, tiller number, filled grain number and unfilled grain number in both conditions, but grain productivity and grain yield were appeared only under drought stress conditions. This indicated the additive effects of genes in controlling of these traits and, therefore, these traits can be improved by selection in breeding programes. There were positive correlations between grain yield and tiller number, panicle exsertion, grain productivity and width and length of flagh leaf in normal irrigation condition. Grain yield had also positive correlation with flagh leaf width, panicle exsertion, filled grain number, grain productivity and width and length of grain under drought stress conditions. Regression and path analyses indicated positive effects of grain width, grain productivity, flagh leaf width, filled grain number, tiller number and internode length in normal irrigation conditions and grain productivity, grain width and tiller number under drought stress conditions. Therefore, it is possible to improve grain yield using these variables. The genotypes including G1, G2, G3 and G4 had the highest values of grain yield $\left(2788,2163,2178\right.$ and $\left.2194 \mathrm{~kg} \mathrm{ha}^{-1}\right)$ under drought stress conditions, and influencing traits on grain yield. Due to the stress index (SI), these genotypes were also as drought tolerant and, thus, can be used for introducing the drought tolerance cultivar.
\end{abstract}

Keywords: Correlation, Heritability, Limited irrigation, Path analysis, Regression, Rice 